\title{
Faster-than-Nyquist Asynchronous NOMA Outperforms Synchronous NOMA
}

\author{
Shuangyang Li, Student Member, IEEE, Zhiqiang Wei, Member, IEEE, Weijie Yuan, Member, IEEE, \\ Jinhong Yuan, Fellow, IEEE, Baoming Bai, Senior Member, IEEE, Derrick Wing Kwan Ng, Fellow, IEEE, and \\ Lajos Hanzo, Fellow, IEEE
}

\begin{abstract}
Faster-than-Nyquist (FTN) signaling aided nonorthogonal multiple access (NOMA) is conceived and its achievable rate is quantified in the presence of random link delays of the different users. We reveal that exploiting the link delays may potentially lead to a signal-to-interference-plus-noise ratio (SINR) gain, while transmitting the data symbols at FTN rates has the potential of increasing the degree-of-freedom (DoF). We then unveil the fundamental trade-off between the SINR and DoF. In particular, at a sufficiently high symbol rate, the SINR gain vanishes while the DoF gain achieves its maximum, where the achievable rate is almost $(1+\beta)$ times higher than that of the conventional synchronous NOMA transmission in the high signal-to-noise ratio (SNR) regime, with $\beta$ being the roll-off factor of the signaling pulse. Our simulation results verify our analysis and demonstrate considerable rate improvements over the conventional power-domain NOMA scheme.
\end{abstract}

Index Terms-NOMA, asynchronous transmission, achievable rate, faster-than-Nyquist signaling

\section{INTRODUCTION}

The escalating number of wireless devices and sensors has inspired exploring efficient multiple access solutions for future Internet-of-Things (IoT) networks. In particular, nonorthogonal multiple access (NOMA) has been extensively studied as a promising radio access scheme due to its superiority in handling massive connectivity [2], which is a crucial requirement for IoT networks. In contrast to conventional orthogonal multiple access (OMA) schemes, NOMA allows more than one user to transmit their information via the same time-frequency resource block, hence enjoying an improved spectral efficiency via exploiting their channel disparities [3]. However, future beyond fifth-generation (B5G) wireless networks are expected to attain even higher spectral efficiency than the 5G networks [4]. Therefore, the existing NOMA schemes have to be improved in order to meet the ultra-high data rate requirement of $\mathrm{B} 5 \mathrm{G}$ wireless networks.

Hence, diverse advanced transmission schemes have been proposed in the literature [5]-[12]. For example, a NOMAbased system design by considering minimum error probability

Part of the paper was presented in the IEEE Wireless Communications and Networking Conference 2021 [1]. This work was supported in part by the National Nature Science Foundation of China under Grant 62171356. D. W. $\mathrm{K}$. Ng is supported by funding from the UNSW Digital Grid Futures Institute, UNSW, Sydney, under a cross-disciplinary fund scheme and by the Australian Research Council's Discovery Project (DP210102169). L. Hanzo would like to acknowledge the financial support of the Engineering and Physical Sciences Research Council projects EP/P034284/1 and EP/P003990/1 (COALESCE) as well as of the European Research Council's Advanced Fellow Grant QuantCom (Grant No. 789028). was conceived in [5], where the effect of realistic imperfect successive interference cancellation (SIC) was also taken into account for the system design. The simulation results showed that a superior error performance can be obtained based on the proposed design compared to the conventional powerdomain NOMA. Furthermore, the rate-splitting multiple access (RSMA) was considered in [6], where each user partially decoded the interference and treated the rest of the interference as noise. In this way, RSMA has been shown, by Clerckx et. al., to achieve both an improved spectral efficiency and an enhanced robustness against imperfect channel state information at the transmitter [7]. Besides, an intelligent reflecting surface (IRS) -assisted NOMA scheme was designed in [8] by Ding et. al., where the IRSs are used for beneficially aligning the cell-edge users' effective channel vectors with the predetermined spatial directions in order to improve the overall performance of NOMA transmissions. Moreover, the sparse code multiple access (SCMA) transmission allows more users to transmit their information among a less number of resource blocks using an appropriately designed codebook [9]. By relying on a carefully constructed codebook, it has been shown that SCMA can achieves a better error performance than conventional power-domain NOMA [9]. On the other hand, asynchronous NOMA (aNOMA) transmissions have also been shown to offer an extended achievable rate region compared to conventional synchronous NOMA transmissions [1], [10], [11]. Specifically, Haci et. al. [10] conceived the aNOMA concept based on orthogonal frequency-division multiplexing (OFDM) and showed that the aNOMA scheme outperforms both the conventional synchronous NOMA scheme and the classic orthogonal frequency-division multiple access (OFDMA) arrangement in terms of both its bit error rate (BER) and capacity. Furthermore, time domain (TD) aNOMA transmission was evaluated by Zou et. al. [11], where the authors considered a fixed link delay introduced by each user and the data was conveyed by finite-duration signaling pulses. Similar to the frequency domain (FD) aNOMA transmission, the TD aNOMA technique has also shown advantages in terms of its achievable rate [11]. Moreover, NOMA transmission based on faster-than-Nyquist (FTN) signaling was also considered in the literature [12]. Indeed, FTN signaling is a classic nonorthogonal signaling scheme exhibiting an enhanced spectral efficiency [13]-[16], where data is transmitted at a symbol rate higher than the Nyquist intersymbol interference (ISI)- 
free rate ${ }^{1}$ [19]. Although FTN-based NOMA (FTN-NOMA) transmission is expected to have an increased achievable rate, its theoretical rate analysis has not been disseminated in the open literature.

As an extension of the state-of-the-art, we improve the conventional power-domain NOMA scheme for bandlimited uplink transmissions from two different perspectives, namely, its signal-to-interference-plus-noise ratio (SINR) and its degreeof-freedom (DoF). In particular, we show that an SINR gain can be achieved by exploiting the link delay difference between different users, while a DoF gain is attained by FTN signaling. However, interestingly enough, there is a fundamental trade-off between the SINR gain and DoF gain. In fact, the potential of both the SINR and DoF improvements arise from the spectral aliasing of signal transmissions under a practical bandwidth constraint. Apart from the practical benefits of an enhanced throughput, the proposed NOMA scheme also provides theoretical insights. It is widely recognized that spectral aliasing occurs when the signal bandwidth is higher than half the sampling frequency. We reveal two ways of mitigating the effect of spectral aliasing. The first one is by imposing phase rotations on the signal spectrum (corresponding to imposing delay for the TD signal), so that the power spectral density (PSD) at the aliased FD components generally has a reduced value due to the superposition of adjacent spectra (caused by spectral aliasing). The second one is to transmit signals at an FTN symbol rate ${ }^{2}$. These two solutions lead to potential SINR and DoF gains, and we will show that there is a tradeoff between these gains.

To further clarify the above arguments, we conceive more complex but practical uplink transmission scenarios in this paper, where each user experiences a random link delay. This is in contrast to the majority of the literature, e.g., [2], [21] and the references therein, which assumed perfectly timesynchronous transmission among NOMA users. However, the perfect synchronization assumption is often unrealistic in practical uplink transmissions, due to the use of different clock generators adopted at geographically distributed uplink users and owing to the distance-dependent propagation delays. As a consequence, the signals from multiple users cannot be synchronously superimposed at the BS as commonly assumed in the literature ${ }^{3}$ [2], [3], [21]. Given practical considerations, we study the achievable rates of both the asynchronous FTNNOMA (aFTN-NOMA) scheme and the aNOMA scheme (aFTN-NOMA scheme using Nyquist symbol rate). We also compare them to the achievable rates of the conventional synchronous power-domain NOMA schemes under the same channel conditions. In summary, our work is motivated by the following facts: 1) Although FTN-NOMA transmission has been proposed in the literature, its transmission with

\footnotetext{
${ }^{1}$ It should be noted that the idea of transmitting symbols faster is not limited to the TD. For example, it has been shown that transmitting symbols faster in the FD also enjoys advantages [17], [18].

${ }^{2}$ It should be noted that the potential DoF gain is not due to the oversampling but owing to the increased symbol rate at the transmitter side. More details on this argument can be found in [20].

${ }^{3}$ Although the conception of the $2 \mathrm{G}$ system's adaptive time-frame alignment has been used, in the face of mobility, non-negligible time-of-arrival differences are experienced at the uplink receiver.
}

TABLE I

SUMMARY OF RELATED WORKS

\begin{tabular}{|l|c|c|c|c|c|}
\hline Related works & {$[1]$} & {$[10]$} & {$[11]$} & {$[12]$} & This work \\
\hline Asynchronous transmission & $\checkmark$ & $\checkmark$ & $\checkmark$ & $\boldsymbol{X}$ & $\checkmark$ \\
\hline Arbitrary link delay & $\checkmark$ & $\boldsymbol{X}$ & $\boldsymbol{X}$ & $\boldsymbol{X}$ & $\checkmark$ \\
\hline FTN signaling & $\boldsymbol{X}$ & $\boldsymbol{X}$ & $\boldsymbol{X}$ & $\checkmark$ & $\checkmark$ \\
\hline Achievable rate analysis & $\checkmark$ & $\checkmark$ & $\checkmark$ & $\boldsymbol{X}$ & $\checkmark$ \\
\hline
\end{tabular}

arbitrary link delay has not been considered, even though it is of great practical interest; 2) The theoretical rate analysis of FTN-NOMA is absent in the literature and the potential rate improvement attained by asynchronous transmissions due to arbitrary link delays is still not well-understood; 3) The fundamental relationships between the symbol rate, asynchronous transmission, and spectrum aliasing has not been unveiled previously. Furthermore, their effects on the achievable rate has not been documented. For a better understanding of our motivations and the novelty of this work, we have summarized the major contributions of the related literature in Table I in comparison to this work. Corresponding to the motivations of this work, the main contributions of this paper are summarized as follows:

- We derive the closed-form expression of the mutual information for aFTN-NOMA schemes at various symbol rates using SIC detection and specific link delays for each user.

- In order to characterize the effect of link delays, we propose to apply bounding techniques to the mutual information derived for each user. Specifically, we show that the link delay may potentially affect the achievable rate via changing the discrete-time Fourier transform (DTFT) of the transmitted signal. Therefore, we derive both the upper- and lower-bounds of the signal DTFTs that are independent from the link delays.

- Based on the DTFT bounds derived, we invoke Szegö's Theorem [22] for characterizing the mutual information of the aFTN-NOMA schemes at various symbol rates and derive the corresponding upper- and lower-bounds of the achievable rates. Based on the bounds derived, the influence of the link delay and symbol rate on the signal spectrum (DTFT) is unveiled. In particular, we also reveal the relationships between the effect of spectral aliasing and the link delay as well as the symbol rate.

- We show that exploiting the link delay actually results in an SINR gain, while increasing the symbol rate may lead to a DoF gain, but there is a trade-off between them. Explicitly, at a sufficiently high symbol rate, the SINR gain vanishes, while the DoF gain attains its maximum. In this case, the achievable rate is essentially the capacity associated with the specific signaling pulse. Explicitly, this is about $1+\beta$ times higher than the conventional synchronous NOMA scheme in the high signal-to-noise ratio (SNR) regime, with $0 \leq \beta \leq 1$ being the FD roll-off factor of the signaling pulse.

- Simulation results confirm the accuracy of our analysis, and demonstrate a significant improvement in terms of the rate attained by the aFTN-NOMA scheme compared 
to the perfectly synchronous NOMA scheme.

Notations: $\max \{\cdot\}$ and $\min \{\cdot\}$ denote the maximization and minimization operations, respectively; $\otimes$ denotes the convolution operation; $I(\cdot ; \cdot)$ and $h(\cdot)$ denote the mutual information and the differential entropy, respectively; $\delta(\cdot)$ denotes the Dirac delta function; $\mathbf{I}_{N \times N}$ denotes the identity matrix of size $N \times N$; the notations $(\cdot)^{\mathrm{T}},(\cdot)^{*}$ represent the transpose and the conjugate operations for a matrix, respectively; the blackboard bold letter $\mathbb{E}[\cdot]$, and $\mathbb{C}$ denote the expectation operator and the complex number field, respectively.

\section{SySTEM MODEL}

Let us consider a pair of single-carrier asynchronous NOMA uplink schemes transmitting over block-fading channels. Specifically, we assume that there are $K$ users and each user transmits $N$ information symbols, i.e., $\mathbf{x}_{k}=$ $\left[x_{k}[0], x_{k}[1], \ldots, x_{k}[N-1]\right]^{\mathrm{T}}$, for $1 \leq k \leq K$. To model the asynchronous transmissions, we assume a random link delay $\tau[k], 1 \leq k \leq K$, for each user. We adopt the common block-fading channel model of NOMA systems. Let $d_{k}$ denote the distance between the $k$-th user and the base station (BS) and $\alpha$ represent the path loss factor. Then, the channel coefficient $h_{k} \in \mathbb{C}, 1 \leq k \leq K$, of the $k$-th user is assumed to be complex-valued Gaussian distributed with a zero mean and variance of $\frac{1}{1+d_{k}^{\alpha}}$ [3], [23]. Let $T$ denote the Nyquist symbol duration. Without loss of generality, we consider the root raised cosine (RRC) pulse having an FD roll-off factor $0 \leq \beta \leq 1$ as our signaling pulse $p(t)$, which is bandlimited, real-valued, and $T$-orthogonal with a normalized energy, i.e., $\int_{-\infty}^{\infty}|p(t)|^{2} \mathrm{~d} t=1$. The Fourier transform of $p(t)$ is denoted by $H_{p}(f)$, which is strictly bandlimited within the frequency interval of $f \in[-W, W]$, with $W \triangleq \frac{1+\beta}{2 T}$ denoting the baseband bandwidth. In what follows, we will present the system models of both NOMA schemes considered. For the ease of presentation, we will slightly abuse the related notations without causing ambiguity.

\section{A. Asynchronous NOMA Scheme}

For the aNOMA scheme, the $k$-th user's transmitted signal is of the following form [1]:

$$
s_{k}(t)=\sqrt{E_{s}[k]} \sum_{n=0}^{N-1} x_{k}[n] p(t-n T),
$$

where $E_{s}[k]$ is the average symbol energy of the $k$-th user. Then, the signal $r(t)$ received by the $\mathrm{BS}$ is given by

$$
\begin{aligned}
r(t) & =\sum_{k=1}^{K} h_{k} s_{k}(t-\tau[k])+w(t) \\
& =\sum_{k=1}^{K} \sum_{n=0}^{N-1} h_{k} \sqrt{E_{s}[k]} x_{k}[n] p(t-n T-\tau[k])+w(t),
\end{aligned}
$$

where $w(t) \in \mathbb{C}$ is the additive white Gaussian noise (AWGN) at the BS with zero mean and one-sided PSD $N_{0}$. For illustrating the asynchronous transmission, we provide a brief diagram in Fig. 1(a), where we consider the uplink transmission of

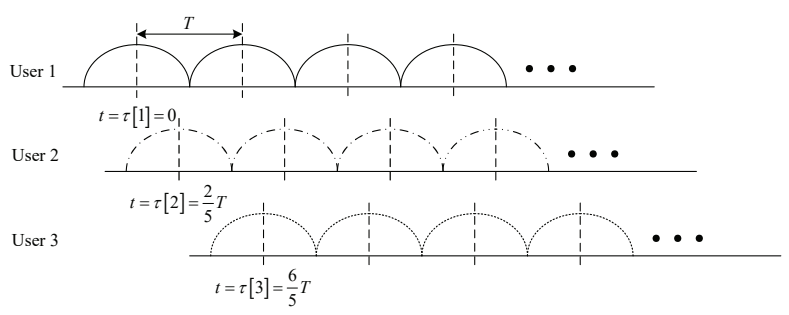

(a) Diagram of aNOMA transmissions, where $K=3$ users are considered and their link delays are $0, \frac{2}{5} T$, and $\frac{6}{5} T$, respectively.

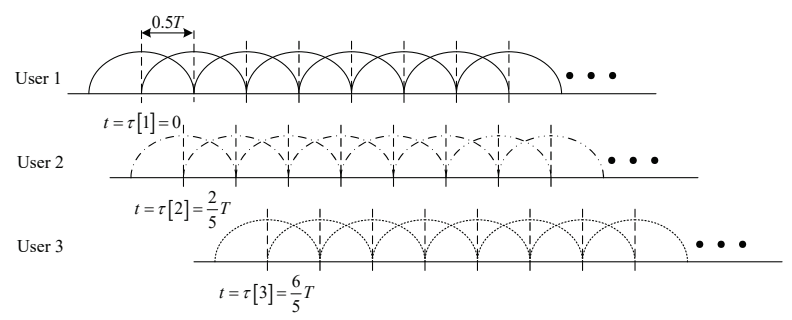

(b) Diagram of aFTN-NOMA transmissions, where $K=3$ users are considered and their link delays are $0, \frac{2}{5} T$, and $\frac{6}{5} T$, respectively.

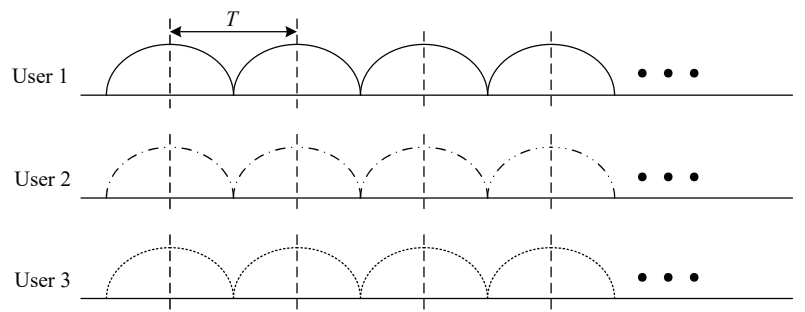

(c) Diagram of NOMA transmissions, where $K=3$ users are considered.

Fig. 1. The uplink transmission diagram of considered scheme, where $K=3$ users are considered.

3 users having the specific link delays of $0, \frac{2}{5} T$, and $\frac{6}{5} T$, respectively.

Observe from the diagram that there is no ISI between the information symbols of a given user due to the $T$ orthogonal property, while each information symbol of the $k$-th user is interfered with all the information symbols of the other users due to the asynchronous transmission. This is the ubiquitous multi-user interference (MUI). By performing matched-filtering and Nyquist rate sampling for $r(t)$, the $n$-th element of the received symbol vector corresponding to the $k$-th user $\mathbf{y}_{k}=\left[y_{k}[0], y_{k}[1], \ldots, y_{k}[N-1]\right]^{\mathrm{T}}$ is given by

$$
\begin{aligned}
y_{k}[n] & =\int_{-\infty}^{\infty} r(t) p^{*}(t-n T-\tau[k]) \mathrm{d} t \\
& =\sum_{l=1}^{K} \sum_{m=0}^{N-1} h_{l} \sqrt{E_{s}[l]} x_{l}[m] g[m-n, \tau[l]-\tau[k]]+\eta_{k}[n] .
\end{aligned}
$$

In (3), the term $g[m-n, \tau[l]-\tau[k]]$ represents the MUI between different users for the aNOMA scheme, which is 


$$
\mathbf{G}_{l, k}=\left[\begin{array}{cccc}
g[0, \tau[l]-\tau[k]] & g[1, \tau[l]-\tau[k]] & \cdots & g[N-1, \tau[l]-\tau[k]] \\
g[-1, \tau[l]-\tau[k]] & g[0, \tau[l]-\tau[k]] & \cdots & g[N-2, \tau[l]-\tau[k]] \\
\vdots & & \ddots & \vdots \\
g[1-N, \tau[l]-\tau[k]] & g[2-N, \tau[l]-\tau[k]] & \cdots & g[0, \tau[l]-\tau[k]]
\end{array}\right]
$$

$$
\tilde{\mathbf{G}}_{l, k}=\left[\begin{array}{cccc}
\tilde{g}_{\zeta}[0, \tau[l]-\tau[k]] & \tilde{g}_{\zeta}[1, \tau[l]-\tau[k]] & \cdots & \tilde{g}_{\zeta}[N-1, \tau[l]-\tau[k]] \\
\tilde{g}_{\zeta}[-1, \tau[l]-\tau[k]] & \tilde{g}_{\zeta}[0, \tau[l]-\tau[k]] & \cdots & \tilde{g}_{\zeta}[N-2, \tau[l]-\tau[k]] \\
\vdots & & \ddots & \vdots \\
\tilde{g}_{\zeta}[1-N, \tau[l]-\tau[k]] & \tilde{g}_{\zeta}[2-N, \tau[l]-\tau[k]] & \cdots & \tilde{g}_{\zeta}[0, \tau[l]-\tau[k]]
\end{array}\right]
$$

given by

$$
\begin{aligned}
g[\Delta k, \Delta \tau] & \triangleq \int_{-\infty}^{\infty} p(t) p^{*}(t+\Delta k T+\Delta \tau) \mathrm{d} t \\
& =\int_{-\infty}^{\infty}\left|H_{p}(f)\right|^{2} \exp (j 2 \pi f(\Delta k T+\Delta \tau)) \mathrm{d} f,
\end{aligned}
$$

where the second equation is due to the Parseval's Theorem. The term $\eta_{k}[n]$ in (3) denotes the corresponding colored noise sample, where $\mathbb{E}\left\{\eta_{k}[n] \eta_{l}^{*}[m]\right\}=N_{0} g[m-n, \tau[l]-\tau[k]]$. For the ease of presentation, (3) can be equivalently expressed in the matrix form of

$$
\mathbf{y}_{k}=\sum_{l=1}^{K} h_{l} \sqrt{E_{s}[l]} \mathbf{G}_{l, k} \mathbf{x}_{l}+\boldsymbol{\eta}_{k},
$$

where $\mathbf{G}_{l, k}$ is the MUI channel matrix of the aNOMA scheme, characterizing the MUI inflicted by the $l$-th user upon the $k$-th user, as shown in (6) at the top of this page. It can be shown that $\mathbf{G}_{l, k}$ is a Toeplitz matrix and we have $\mathbf{G}_{k, k}=\mathbf{I}_{N \times N}$ for $1 \leq k \leq K$. Meanwhile, the noise vector $\boldsymbol{\eta}_{k}$ is given by $\boldsymbol{\eta}_{k}=\left[\eta_{k}[0], \eta_{k}[1], \ldots, \eta_{k}[N-1]\right]^{\mathrm{T}}$, for $1 \leq k \leq K$, and it can be shown that $\mathbb{E}\left\{\eta_{k}[m] \eta_{k}^{*}[n]\right\}=N_{0}$, for $1 \leq k \leq K$ and $0 \leq n, m \leq N-1$.

\section{B. Asynchronous FTN-NOMA Scheme}

For the aFTN-NOMA scheme, the $k$-th user's transmitted signal is given by

$$
\tilde{s}_{k}(t)=\sqrt{E_{s}[k]} \sum_{n=0}^{N-1} x_{k}[n] p(t-n \zeta T),
$$

where $\zeta \in[0,1]$ denotes the TD compression factor and the symbol period of the aFTN-NOMA scheme is $\zeta T$ [24], [25]. Similar to the previous subsection, the signal $\tilde{r}(t)$ received by the $\mathrm{BS}$ is given by

$$
\begin{aligned}
\tilde{r}(t) & =\sum_{k=1}^{K} h_{k} \tilde{s}_{k}(t-\tau[k])+w(t) \\
& =\sum_{k=1}^{K} \sum_{n=0}^{N-1} h_{k} \sqrt{E_{s}[k]} x_{k}[n] p(t-n \zeta T-\tau[k])+w(t) .
\end{aligned}
$$

The transmission diagram of the aFTN-NOMA scheme having $\zeta=0.5$ is shown in Fig. 1(b), where 3 users having specific link delays $0, \frac{2}{5} T$, and $\frac{6}{5} T$ are considered. In contrast to Fig. 1(a), each information symbol is interfered by all the other information symbols among all users owing to both the FTN symbol rate and the asynchronous transmission. For matched-filtering and FTN-rate sampling, the $n$-th element of the received symbol vector corresponding to the $k$-th user $\mathbf{y}_{k}=\left[y_{k}[0], y_{k}[1], \ldots, y_{k}[N-1]\right]^{\mathrm{T}}$ is given by

$$
\begin{aligned}
y_{k}[n]= & \int_{-\infty}^{\infty} \tilde{r}(t) p^{*}(t-n \zeta T-\tau[k]) \mathrm{d} t \\
= & \sum_{l=1}^{K} \sum_{m=0}^{N-1} h_{l} \sqrt{E_{s}[l]} x_{l}[m] \tilde{g}_{\zeta}[m-n, \tau[l]-\tau[k]] \\
& +\tilde{\eta}_{k}[n] .
\end{aligned}
$$

In (9), the term $\tilde{g}_{\zeta}[\Delta k, \Delta \tau]$ represents the MUI between different information symbols given by

$$
\begin{aligned}
\tilde{g}_{\zeta}[\Delta k, \Delta \tau] & \triangleq \int_{-\infty}^{\infty} p(t) p^{*}(t+\Delta k \zeta T+\Delta \tau) \mathrm{d} t \\
& =\int_{-\infty}^{\infty}\left|H_{p}(f)\right|^{2} \exp (j 2 \pi f(\Delta k \zeta T+\Delta \tau)) \mathrm{d} f .
\end{aligned}
$$

The term $\tilde{\eta}_{k}[n]$ in (9) denotes the corresponding colored noise sample, where $\mathbb{E}\left\{\tilde{\eta}_{k}[n] \tilde{\eta}_{l}^{*}[m]\right\}=N_{0} \tilde{g}_{\zeta}[m-n, \tau[l]-\tau[k]]$. Similar to the previous subsection, we consider the equivalent matrix expression of (9), i.e.,

$$
\mathbf{y}_{k}=\sum_{l=1}^{K} h_{l} \sqrt{E_{s}[l]} \tilde{\mathbf{G}}_{l, k} \mathbf{x}_{l}+\tilde{\boldsymbol{\eta}}_{k},
$$

where $\tilde{\mathbf{G}}_{l, k}$ is the MUI channel matrix for the aFTN-NOMA scheme, characterizing the interference inflicted by the $l$-th user on the $k$-th user, as shown in (12) at the top of this page. Again, $\tilde{\mathbf{G}}_{l, k}$ is a Toeplitz matrix and the noise vector $\tilde{\boldsymbol{\eta}}_{k}$ is given by $\tilde{\boldsymbol{\eta}}_{k}=\left[\tilde{\eta}_{k}[0], \tilde{\eta}_{k}[1], \ldots, \tilde{\eta}_{k}[N-1]\right]^{\mathrm{T}}$, where $\mathbb{E}\left\{\tilde{\boldsymbol{\eta}}_{k} \tilde{\boldsymbol{\eta}}_{k}^{H}\right\}=N_{0} \widetilde{\mathbf{G}}_{k, k}$. 


$$
\begin{aligned}
& I_{\mathbf{h}, \boldsymbol{\tau}, \zeta}\left(\mathbf{y}_{k} ; \mathbf{x}_{k} \mid \mathbf{x}_{1}, \ldots, \mathbf{x}_{k-1}\right) \\
= & \frac{1}{2} \log _{2} \operatorname{det}\left[\mathbf{I}_{N \times N}+\frac{\left|h_{k}\right|^{2} E_{s}[k] \tilde{\mathbf{G}}_{k, k} \tilde{\mathbf{G}}_{k, k}^{\mathrm{T}}}{N_{0}}\left(\tilde{\mathbf{G}}_{k, k}+\frac{\sum_{l=k+1}^{K}\left|h_{l}\right|^{2} E_{s}[l]}{N_{0}} \tilde{\mathbf{G}}_{l, k} \tilde{\mathbf{G}}_{l, k}^{\mathrm{T}}\right)^{-1}\right] .
\end{aligned}
$$

\section{Connections to the Conventional Synchronous NOMA Scheme}

Conventionally, the impact of link delay difference is assumed to be perfectly eliminated by adaptive time frame alignment schemes at the BS [3], [26] for synchronous NOMA transmission. Consequently, the signals transmitted from different users are perfectly aligned with each other at the BS, as shown in Fig. 1(c). By comparing aNOMA, aFTN-NOMA, and conventional synchronous NOMA schemes, it is plausible that the aFTN-NOMA scheme is the most general scheme. More specifically, when the compression factor is $\zeta=1$, the aFTN-NOMA scheme degenerates to the aNOMA scheme. When there is no link delay difference among the users, the aNOMA scheme degenerates to the conventional synchronous NOMA scheme.

On the other hand, we notice that both the aNOMA and aFTN-NOMA schemes have at most $K N$ received symbols at the BS, while the conventional synchronous NOMA only has $N$ received symbols. Furthermore, we also notice that the aNOMA, aFTN-NOMA and conventional synchronous NOMA schemes generally occupy different time resources. Let $\tau_{\max }=\max \{\tau[1], \tau[2], \ldots, \tau[K]\}$ be the maximum link delay, which is usually negligible compared to the frame duration of the signals transmitted in practical systems [26], [27]. To support each user transmitting $N$ information symbols, the conventional synchronous NOMA scheme roughly requires $N T$ seconds for its transmission, aNOMA scheme requires $N T+\tau_{\max }$ seconds, while the aFTN-NOMA scheme only requires $N \zeta T+\tau_{\max }$ [28], [29].

In the following, we investigate the achievable rates of both the aNOMA and aFTN-NOMA schemes based on (5) and (11).

\section{Achievable Rate Analysis}

In this section, we focus our attention on the achievable rates of both the aNOMA and aFTN-NOMA schemes. We will first derive the closed-form expression of the mutual information for the uplink transmission and then apply Szegö's Theorem [22], [30] to obtain further important insights. Particularly, Szegö's Theorem is closely related to the DTFT of the underlying Toeplitz coefficients. Therefore, we will also investigate the characteristics of the DTFT with respect to the link delay and symbol rate. For the ease of derivation, we assume that the elements in the transmitted symbol vector $\mathbf{x}_{k}$ are independent and identically distributed (i.i.d.) complex Gaussian variables with average symbol energy $E_{s}[k]$, for $\forall k$, $1 \leq k \leq K$. Since the aNOMA scheme can be viewed as a special case of the aFTN-NOMA scheme with $\zeta=1$, we will commence with the analysis of the aFTN-NOMA scheme.
Without loss of generality, let us assume that the channel coefficients are sorted in descending order, i.e., $\left|h_{1}\right|^{2} \geq\left|h_{2}\right|^{2} \geq$ $\ldots \geq\left|h_{K}\right|^{2}$. Conventionally, SIC detection is applied at the BS for general NOMA systems ${ }^{4}$ [3]. To analyze the achievable rates, it is commonly assumed that the MUI introduced by users $1,2, \ldots, k-1$ is perfectly cancelled for the detection of the $k$-th user [3]. Therefore, the asymptotic instantaneous achievable rate for the $k$-th user under SIC detection is given by

$$
\begin{array}{r}
R_{\mathbf{h}, \boldsymbol{\tau}, \zeta}^{k} \triangleq \lim _{N \rightarrow \infty} \frac{1}{N} I_{\mathbf{h}, \boldsymbol{\tau}, \zeta}\left(\mathbf{y}_{k} ; \mathbf{x}_{k} \mid \mathbf{x}_{1}, \ldots, \mathbf{x}_{k-1}\right) \\
\text { bits per channel use }
\end{array}
$$

In particular, the closed-form expression of $I_{\mathbf{h}, \boldsymbol{\tau}, \zeta}\left(\mathbf{y}_{k} ; \mathbf{x}_{k} \mid \mathbf{x}_{1}, \ldots, \mathbf{x}_{k-1}\right)$ is formulated in the following lemma.

Lemma 1 (Conditional Mutual Information for SIC Detection): For SIC detection, the conditional mutual information $I_{\mathbf{h}, \boldsymbol{\tau}, \zeta}\left(\mathbf{y}_{k} ; \mathbf{x}_{k} \mid \mathbf{x}_{1}, \ldots, \mathbf{x}_{k-1}\right)$ of the aFTN-NOMA scheme is given in (14) at the top of this page.

Proof: The proof is given in Appendix A.

The above equation is essentially the mutual information calculation over the channel with colored Gaussian noise, where the covariance matrix of the noise samples plus the interference is given by $N_{0} \tilde{\mathbf{G}}_{k, k}+\sum_{l=k+1}^{K}\left|h_{l}\right|^{2} E_{s}[l] \tilde{\mathbf{G}}_{l, k} \tilde{\mathbf{G}}_{l, k}^{\mathrm{T}}$. It can be observed from Lemma 1 that due to the link delay and the symbol rate of each user, the corresponding interference term of the aFTN-NOMA systems for the $k$-th user is different from that of the conventional synchronous NOMA systems. With the help of Lemma 1, we now proceed to analyze the asymptotic instantaneous achievable rate $R_{\mathbf{h}, \boldsymbol{\tau}, \zeta}^{k}$ by invoking Szegö's Theorem in order to obtain further important insights. For reference, Szegö's Theorem is stated as follows.

Lemma 2 (Szegö's Theorem [22], [30]): Let V denote a size $N \times N$ positive definite Toeplitz matrix $\mathbf{V}$, i.e.,

$$
\mathbf{V}=\left[\begin{array}{cccc}
v_{0} & v_{1} & \cdots & v_{N-1} \\
v_{-1} & v_{0} & \cdots & v_{N-2} \\
\vdots & & \ddots & \vdots \\
v_{1-N} & v_{2-N} & \cdots & v_{0}
\end{array}\right]
$$

whose eigenvalues are given by $\left\{\lambda_{0}, \lambda_{1}, \ldots \lambda_{N-1}\right\}$. Then, for an arbitrary continuous function $f_{c}(\cdot)$, we have

$$
\lim _{N \rightarrow \infty} \frac{1}{N} \sum_{n=0}^{N-1} f_{c}\left(\lambda_{n}\right)=\frac{1}{2 \pi} \int_{-\pi}^{\pi} f_{c}(V(\omega)) \mathrm{d} \omega
$$

${ }^{4}$ We note that some lattice-coding-based approaches can be applied to replace the SIC detection [31], [32]. 


$$
\left|H_{p}(f)\right|^{2}= \begin{cases}T, & |f|<(1-\beta) /(2 T) \\ T \cos ^{2}\left(\frac{\pi T}{2 \beta}\left(|f|-\frac{1-\beta}{2 T}\right)\right), & (1-\beta) /(2 T) \leq|f| \leq(1+\beta) /(2 T) \\ 0, & |f|>(1+\beta) /(2 T)\end{cases}
$$

where $V(\omega)$ is the corresponding DTFT of the Toeplitz coefficients $\left\{\ldots, v_{-2}, v_{-1}, v_{0}, v_{1}, v_{2}, \ldots\right\}$, and it is given by

$$
V(\omega)=\sum_{k=-\infty}^{\infty} v_{k} e^{-j k \omega} .
$$

Szegö's Theorem is a classic tool eminently suitable for analyzing the determinant of Toeplitz matrices, which is rooted in the FD characteristics of the Toeplitz coefficients, i.e., the DTFT. In particular, DTFT analysis has been widely applied in the research of signal sampling, which describes the FD representation of the underlying samples. Let us define $\mathbf{T}_{l, k} \triangleq$ $\tilde{\mathbf{G}}_{l, k} \tilde{\mathbf{G}}_{l, k}^{\mathrm{T}}$ and

$$
\begin{aligned}
& \mathbf{P}_{k} \triangleq \mathbf{I}_{N \times N}+\frac{\left|h_{k}\right|^{2} E_{s}[k] \mathbf{T}_{k, k}}{N_{0}} \\
& \left(\tilde{\mathbf{G}}_{k, k}+\frac{\sum_{l=k+1}^{K}\left|h_{l}\right|^{2} E_{s}[l]}{N_{0}} \mathbf{T}_{l, k}\right)^{-1}
\end{aligned}
$$

To apply Szegö's Theorem, we first have to verify that $\mathbf{P}_{k}$ is a positive definite Toeplitz matrix in the asymptotic regime, i.e., $N \rightarrow \infty$, for any $1 \leq k \leq K$. Specifically, we have the following lemma.

Lemma 3 (Positive Definiteness of the Asymptotical Toeplitz Matrix): As $N \rightarrow \infty$, both $\tilde{\mathbf{G}}_{l, k}$ and $\mathbf{T}_{l, k}$ are asymptotically positive definite Toeplitz matrices for any $1 \leq k \leq K$. Furthermore, $\mathbf{P}_{k}$ is also an asymptotically positive definite Toeplitz matrix for $1 \leq k \leq K$ as $N \rightarrow \infty$.

Proof: The proof is given in Appendix B.

Next, we apply Szegö's Theorem to (14). The application of Szegö's Theorem requires the derivation of the DTFT of the Toeplitz coefficients. As shown in (14), there are two types of Toeplitz matrices, namely, $\tilde{\mathbf{G}}_{l, k}$ and $\mathbf{T}_{l, k}$. In particular, the Toeplitz coefficients of $\tilde{\mathbf{G}}_{l, k}$ are given by $\left\{\tilde{g}_{\zeta}[n, \tau[l]-\tau[k]]\right\}$. On the other hand, it can be shown that in the asymptotic regime, the Toeplitz coefficients $\left\{t_{l, k}[n]\right\}$ of the asymptotical Toeplitz matrix $\mathbf{T}_{l, k}$ are given by

$$
t_{l, k}[n]=\sum_{m=-\infty}^{\infty} \tilde{g}_{\zeta}[m, \tau[l]-\tau[k]] \tilde{g}_{\zeta}[m-n, \tau[l]-\tau[k]] .
$$

Correspondingly, the DTFTs of the Toeplitz coefficients $\left\{\tilde{g}_{\zeta}[n, \tau[l]-\tau[k]]\right\}$ and $\left\{t_{l, k}[n]\right\}$ are given by

$$
\tilde{G}_{l, k}(2 \pi f \zeta T)=\sum_{n=-\infty}^{\infty} \tilde{g}_{\zeta}[n, \tau[l]-\tau[k]] e^{-j 2 \pi n \zeta T f},
$$

and

$$
\begin{aligned}
& \tilde{T}_{l, k}(2 \pi f \zeta T)=\sum_{n=-\infty}^{\infty} \sum_{m=-\infty}^{\infty} \tilde{g}_{\zeta}[m, \tau[l]-\tau[k]] \\
& \tilde{g}_{\zeta}[m-n, \tau[l]-\tau[k]] e^{-j 2 \pi n \zeta T f},
\end{aligned}
$$

respectively, where $\omega \triangleq 2 \pi f \zeta T$. We note that (20) and (21) depend on both the difference between the link delays of each user and the compression factor $\zeta$. However, the link delays' difference is time-variant and it is therefore generally intractable. As an alternative, we apply bounding techniques to the DTFTs to facilitate the achievable rate analysis. In particular, the bounds of DTFT depend on the corresponding spectra of the signaling pulse adopted. Specifically, the spectrum of the RRC pulse with a roll-off factor $0 \leq \beta \leq 1$ is given in (22) at the top of this page. As a building block for our rate analysis, we consider three FD signals related to $\left|H_{p}(f)\right|^{2}$ and the symbol rate $1 / \zeta T$.

Definition 1 (Folded-Spectrum): Given the symbol rate $1 / \zeta T$ and the underlying signaling pulse spectrum $\left|H_{p}(f)\right|^{2}$, the folded-spectrum is defined by

$$
\left|H_{\text {fo }}(f)\right|^{2} \triangleq \sum_{k=-\infty}^{\infty}\left|H_{p}\left(f-\frac{k}{\zeta T}\right)\right|^{2}
$$

for $f \in\left[-\frac{1}{2 \zeta T}, \frac{1}{2 \zeta T}\right]$ and zero otherwise.

Definition 2 (Twisted Folded-Spectrum): Given the symbol rate $1 / \zeta T$ and the underlying signaling pulse spectrum $\left|H_{p}(f)\right|^{2}$, the twisted folded-spectrum is defined by

$$
\left|H_{\mathrm{tfo}}(f)\right|^{2} \triangleq\left|H_{p}(f)\right|^{2}-\sum_{\substack{k=-\infty \\ k \neq 0}}^{\infty}\left|H_{p}\left(f-\frac{k}{\zeta T}\right)\right|^{2},
$$

for $f \in\left[-\frac{1}{2 \zeta T}, \frac{1}{2 \zeta T}\right]$ and zero otherwise.

Definition 3 (Interference-Reducing-Spectrum): Given the symbol rate $\zeta T$ and the underlying signaling pulse spectrum $\left|H_{p}(f)\right|^{2}$, the interference-reducing-spectrum is defined by

$$
\rho(f) \triangleq \frac{\left|H_{\mathrm{tfo}}(f)\right|^{2}}{\left|H_{\mathrm{fo}}(f)\right|^{2}},
$$

for $f \in\left[-\frac{1}{2 \zeta T}, \frac{1}{2 \zeta T}\right]$ and zero otherwise.

Indeed, the folded-spectrum is commonly considered in the literature of faster-than-Nyquist signaling [33], [34] for the associated capacity analysis. The folded-spectrum indicates that the frequency components outside the interval $\left[-\frac{1}{2 \zeta T}, \frac{1}{2 \zeta T}\right]$ are "folded-in" the interval to form an equivalent FD representation of the transmitted signal. In particular, we refer to this folding effect as "spectral aliasing". Spectral aliasing occurs, when the symbol rate is lower than twice the bandwidth of the signaling pulse, i.e., $\frac{1}{2 \zeta T}<W$. On the other hand, the twisted folded-spectrum can be viewed as a phaserotated version of the folded-spectrum, which is useful for characterizing the FD phase rotation corresponding to the time delay. Furthermore, the interference-reducing-spectrum is the ratio between the aforementioned two spectra, which can be viewed as an indicator of how substantially the interference power is reduced due to the phase-rotation. Noticing that the 
RRC pulse is strictly bandlimited within the frequency interval of $f \in[-W, W]$, it can be shown that $\left|H_{\text {fo }}(f)\right|^{2}$ becomes the spectrum of the sinc pulse $\left|H_{\text {sinc }}(f)\right|^{2}$, i.e., the RRC pulse associated with $\beta=0$, when $\zeta=1$. On the other hand, both $\left|H_{\text {fo }}(f)\right|^{2}$ and $\left|H_{\text {tfo }}(f)\right|^{2}$ become the exact RRC spectrum $\left|H_{p}(f)\right|^{2}$, when $\zeta \leq 1 /(1+\beta)$. For reference, the plots of the folded-spectrum and twisted folded-spectrum for $\beta=0.5, \zeta=1$ and $\beta=0.5, \zeta<2 / 3$ are provided in Fig. 2(a) and Fig. 2(b), respectively. Furthermore, it may be readily seen that upon reducing $\zeta$, the value of the interferencereducing-spectrum $\rho(f)$ tends to 1 for $f \in\left[-\frac{1}{2 \zeta T}, \frac{1}{2 \zeta T}\right]$. In particular, we have $\rho(f)=1$ for $f \in\left[-\frac{1}{2 \zeta T}, \frac{1}{2 \zeta T}\right]$, when $\zeta \leq 1 /(1+\beta)$. This interesting fact actually indicates that when the symbol rate is sufficiently high, the impact of link delay tends to vanish as will be discussed in Section III-B. We note that the folded-spectrum, the twisted folded-spectrum and the interference-reducing-spectrum are important for our analysis, because they are related to the signaling pulse, but they are affected differently with respected to the symbol rate $\frac{1}{\zeta T}$. In the following lemma, we will unveil the intricate relationship between the inverse Fourier series corresponding to $\left|H_{p}(f)\right|^{2}$ and the aforementioned spectra.

Lemma 4 (Bounds on the Infinite Fourier Series): Let $\gamma$ be an arbitrary constant number. Then, within the frequency interval $f \in\left[-\frac{1}{2 \zeta T}, \frac{1}{2 \zeta T}\right]$, the infinite series $\sum_{k=-\infty}^{\infty}\left|H_{p}\left(f-\frac{k}{\zeta T}\right)\right|^{2} e^{-j 2 \pi \gamma k}$ can be upper-bounded and lower-bounded by

$$
\left|H_{\text {tfo }}(f)\right|^{2} \leq \sum_{k=-\infty}^{\infty}\left|H_{p}\left(f-\frac{k}{\zeta T}\right)\right|^{2} e^{-j 2 \pi \gamma k} \leq\left|H_{\text {fo }}(f)\right|^{2},
$$

where the bounds in (26) become exact if $\beta=0$, i.e., $p(t)$ is the sinc pulse, or $\zeta \leq \frac{1}{1+\beta}$. Meanwhile, the upper-bound also becomes exact if $\gamma=0$.

Proof: The proof is given in Appendix C.

Lemma 4 characterizes the effect of phase-rotation on the folded-spectrum, showing that the phase-rotation may potentially change the shape of the spectrum. Observe that the effect of spectral aliasing vanishes for $\beta=0$ or $\zeta \leq \frac{1}{1+\beta}$. Therefore, Lemma 4 implies that the change due to the phase-rotation will no longer exist, when there is no spectral aliasing. This is not unexpected, because when the spectra are sufficiently separated in the FD, only the spectrum corresponding to $k=0$, i.e., $\left|H_{p}(f)\right|^{2}$, has non-zero values in the frequency interval $f \in\left[-\frac{1}{2 \zeta T}, \frac{1}{2 \zeta T}\right]$. In this case, the infinite series $\sum_{k=-\infty}^{\infty}\left|H_{p}\left(f-\frac{k}{\zeta T}\right)\right|^{2} e^{-j 2 \pi \gamma k}$ reduces to $\left|H_{p}(f)\right|^{2}$, which is independent of the phase-rotation. Therefore, introducing phase-rotations (link delay) can no longer affect the value of the folded-spectrum, if there is no spectral aliasing. Given Lemma 4, the DTFTs with respect to (20) and (21) can be upper- and lower-bounded for our analysis, as shown in the following theorems.

Theorem 1 (Bounds on DTFT $\tilde{G}_{l, k}(2 \pi f \zeta T)$ ): Given an arbitrary link delay difference $\Delta \tau \triangleq \tau[l]-\tau[k]$, the DTFT

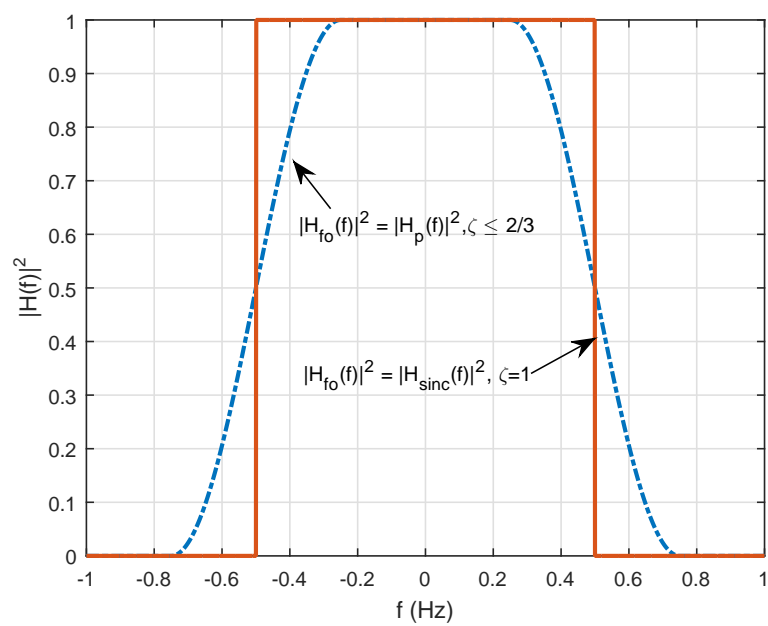

(a) $\left|H_{\text {fo }}(f)\right|^{2}$ for $\beta=0.5, \zeta=1$ and $\beta=0.5, \zeta \leq 2 / 3$.

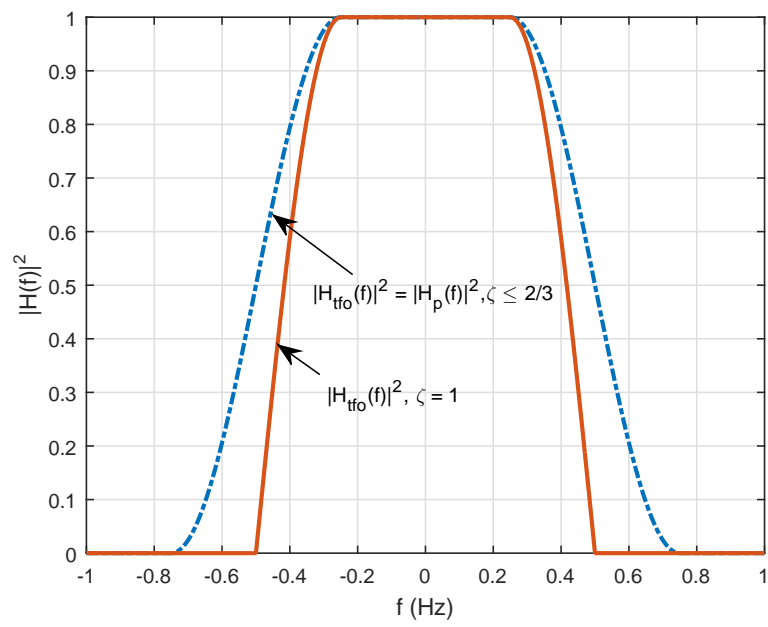

(b) $\left|H_{\mathrm{tfo}}(f)\right|^{2}$ for $\beta=0.5, \zeta=1$ and $\beta=0.5, \zeta \leq 2 / 3$.

Fig. 2. Folded-spectrum and twisted folded-spectrum for $\beta=0.5, \zeta=1$ and $\beta=0.5, \zeta<2 / 3$, where $T=1$.

$\tilde{G}_{l, k}(2 \pi f \zeta T)$ of the form (20) can be upper- and lowerbounded by

$$
\frac{1}{\zeta T}\left|H_{\mathrm{tfo}}(f)\right|^{2} \leq \tilde{G}_{l, k}(2 \pi f \zeta T) \leq \frac{1}{\zeta T}\left|H_{\mathrm{fo}}(f)\right|^{2},
$$

where the bounds in (27) become exact if $\beta=0$, i.e., when $p(t)$ is the sinc pulse, or $\zeta \leq \frac{1}{1+\beta}$. Meanwhile, the upperbound also becomes exact if $\Delta \tau=0$, e.g., $l=k$.

Proof: The proof is given in Appendix D.

Theorem 2 (Bounds on DTFT $\tilde{T}_{l, k}(2 \pi f \zeta T)$ ): Given an arbitrary link delay difference $\Delta \tau \triangleq \tau[l]-\tau[k]$, the DTFT $\tilde{T}_{l, k}(2 \pi f \zeta T)$ of the form (21) can be upper- and lowerbounded by

$$
\left(\frac{1}{\zeta T}\left|H_{\mathrm{tfo}}(f)\right|^{2}\right)^{2} \leq \tilde{T}_{l, k}(2 \pi f \zeta T) \leq\left(\frac{1}{\zeta T}\left|H_{\mathrm{fo}}(f)\right|^{2}\right)^{2},
$$

where the bounds in (27) become exact if $\beta=0$, i.e., if $p(t)$ is the sinc pulse, or $\zeta \leq \frac{1}{1+\beta}$. Meanwhile, the upper-bound also becomes exact if $\Delta \tau=0$, e.g., $l=k$. 
Proof: The proof is given in Appendix E.

Based on the bounds on the DTFTs of the Toeplitz coefficients, we are ready to derive the bounds for the achievable rates. In particular, the main result is given in the following theorem.

Theorem 3 (Bounds on the Achievable Rates of aFTNNOMA Scheme): Let $P_{k} \triangleq \frac{E_{s}[k]}{\zeta T}$ denote the transmit power of the $k$-th user. Then, the asymptotic instantaneous achievable rate of aFTN-NOMA for the $k$-th user $R_{\mathbf{h}, \boldsymbol{\tau}, \zeta}^{k}$ under SIC detection is lower-bounded by

$$
\begin{aligned}
& R_{\mathbf{h}, \boldsymbol{\tau}, \zeta}^{k} \\
& \geq \frac{1}{2 W} \int_{-\frac{1}{2 \zeta T}}^{\frac{1}{2 \zeta T}} \log _{2}\left(1+\frac{\left|h_{k}\right|^{2} P_{k}\left|H_{\mathrm{fo}}(f)\right|^{2}}{N_{0}+\sum_{l=k+1}^{K}\left|h_{l}\right|^{2} P_{l}\left|H_{\mathrm{fo}}(f)\right|^{2}}\right) \mathrm{d} f \\
& \text { bits/s/Hz, (29) }
\end{aligned}
$$

and upper-bounded by

$$
\begin{aligned}
& R_{\mathbf{h}, \boldsymbol{\tau}, \zeta}^{k} \\
& \leq \frac{1}{2 W} \int_{-\frac{1}{2 \zeta T}}^{\frac{1}{2 \zeta T}} \log _{2}\left(1+\frac{\left|h_{k}\right|^{2} P_{k}\left|H_{\mathrm{fo}}(f)\right|^{2}}{N_{0}+\sum_{l=k+1}^{K}\left|h_{l}\right|^{2} P_{l}\left|H_{\mathrm{tfo}}(f)\right|^{2} \rho(f)}\right) \mathrm{d} f \\
& \text { bits/s/Hz. (30) }
\end{aligned}
$$

Proof: The proof is given in Appendix F.

With the help of Theorem 3, we now investigate the achievable rates of both the aNOMA and aFTN-NOMA schemes.

\section{A. Achievable Rates of Asynchronous NOMA Schemes}

For the aNOMA scheme, we have $\zeta=1$, where the foldedspectrum satisfies $\left|H_{\text {fo }}(f)\right|^{2}=T$ within the frequency interval $f \in\left[-\frac{1}{2 T}, \frac{1}{2 T}\right]$. Based on Theorem 3 , the following corollary summarizes the bounds of the achievable rate for aNOMA schemes.

Corollary 1 (Bounds on the Normalized Achievable Rates of aNOMA Scheme): The normalized asymptotic instantaneous achievable rate $R_{\mathbf{h}, \boldsymbol{\tau}, \zeta=1}^{k}$ of the aNOMA scheme for the $k$-th user under SIC detection is lower-bounded by

$$
R_{\mathbf{h}, \boldsymbol{\tau}, \zeta=1}^{k} \geq \frac{1}{2 W T} \log _{2}\left(1+\frac{\left|h_{k}\right|^{2} P_{k} T}{N_{0}+\sum_{l=k+1}^{K}\left|h_{l}\right|^{2} P_{l} T}\right)
$$

and upper-bounded by

$$
\leq \frac{1}{2 W} \int_{-\frac{1}{2 T}}^{\frac{1}{2 T}} \log _{2}\left(1+\frac{\left|h_{k}\right|^{2} P_{k} T}{N_{0}+\sum_{l=k+1}^{K}\left|h_{l}\right|^{2} P_{l}\left|H_{\mathrm{tfo}}(f)\right|^{2} \rho(f)}\right) \mathrm{d} f
$$

Proof: The corollary is a straightforward extension of Theorem 3 and thus the proof is omitted here.

According to Corollary 1, some interesting observations and insights can be revealed.

- Recalling Lemma 4, we observe that both the upperbound and the lower-bound in Corollary 1 are achievable. Theoretically, the upper-bound can be achieved if the signaling pulse's FD roll-off factor is $\beta=0$, i.e., the sinc pulse, while the lower-bound can be achieved, if all the users share the same link delay, corresponding to the conventional synchronous NOMA system.

- For practical RRC pulses, i.e., $\beta \neq 0$, the asynchronous transmission leads to an improved achievable rate region compared to that of the conventional synchronous NOMA systems. Moreover, the upper-bound indicates that the potential data rate improvement of aNOMA systems is due to its reduced MUI energy, yielding an SINR improvement, which is the result of different link delays. An intuitive explanation of this observation is that the aNOMA system naturally avoids the full superposition of maximum MUI owing to the diverse link delays. Hence, aNOMA system is unlikely to suffer from the peak interference energy at each sampling instant, which is consistent with the observations in Fig. 1(a). However, for the sinc pulse, i.e., $\beta=0$, the asynchronous transmission does not provide any rate improvement. This is because the symbol rate is consistent with the bandwidth of the sinc pulse. Hence, no spectral aliasing occurs and thus the introduction of phase-rotations cannot improve the achievable rate. This observation indicates that compared to conventional synchronous NOMA systems, the aNOMA system suffers from less severe MUI caused by the different link delays and offers the potential of achieving higher rates.

- The instantaneous achievable rate region of the aNOMA system is directly determined by both the folded-spectrum and the twisted folded-spectrum. In particular, the achievable rate improvement due to the asynchronous transmission increases upon increasing $\beta$, since the corresponding twisted folded-spectrum has a low energy in the frequency interval $f \in\left[-\frac{1}{2 T}, \frac{1}{2 T}\right]$. However, compared to the zero excess bandwidth of $\beta=0$, the normalized achievable rates of both the aNOMA scheme and the conventional synchronous NOMA scheme are reduced upon increasing of $\beta$ due to the normalization.

\section{B. Achievable Rates of Asynchronous FTN-NOMA Schemes}

We have already formulated the achievable rates of the aFTN-NOMA schemes in Theorem 3. In particular, for $\zeta<1$, we observe that the aFTN-NOMA scheme enjoys both an SINR gain granted by the asynchronous transmissions (corresponding to the interference-reducing-spectrum) and a DoF gain introduced by the FTN transmission (corresponding to the integral range). To further explain advantages of aFTNNOMA, let use formally define the SINR gain and DoF gain of aFTN-NOMA schemes as follows. 
Definition 4 (SINR Gain over Synchronous Transmission): The SINR gain of aFTN-NOMA schemes over the synchronous schemes having the same symbol rate is defined by

$$
\begin{aligned}
\operatorname{Gain}_{\text {SINR }} & \triangleq \frac{\int_{-\frac{1}{2 \zeta T}}^{\frac{1}{2 \zeta T}} \frac{\left|h_{k}\right|^{2} P_{k}\left|H_{\mathrm{fo}}(f)\right|^{2}}{N_{0}+\sum_{l=k+1}^{K}\left|h_{l}\right|^{2} P_{l}\left|H_{\mathrm{tfo}}(f)\right|^{2} \rho(f)} \mathrm{d} f}{\int_{-\frac{1}{2 \zeta T}}^{\frac{1}{2 \zeta T}} \frac{\left|h_{k}\right|^{2} P_{k}\left|H_{\mathrm{fo}}(f)\right|^{2}}{N_{0}+\sum_{l=k+1}^{K}\left|h_{l}\right|^{2} P_{l}\left|H_{\mathrm{fo}}(f)\right|^{2}} \mathrm{~d} f} \\
& =\int_{-\frac{1}{2 \zeta T}}^{\frac{1}{2 \zeta T}} \frac{N_{0}\left|H_{\mathrm{fo}}(f)\right|^{2}+\sum_{l=k+1}^{K}\left|h_{l}\right|^{2} P_{l}\left|H_{\mathrm{fo}}(f)\right|^{4}}{N_{0}\left|H_{\mathrm{fo}}(f)\right|^{2}+\sum_{l=k+1}^{K}\left|h_{l}\right|^{2} P_{l}\left|H_{\mathrm{tfo}}(f)\right|^{4}} \mathrm{~d} f .
\end{aligned}
$$

Definition 5 (DoF Gain over Conventional NOMA): Let us define the effective bandwidth $\mathrm{BW}_{\text {eff }}\left(\frac{1}{\zeta T}\right)$ with respect to the symbol rate $\frac{1}{\zeta T}$ as the frequency interval, where both the folded-spectrum and the twisted folded-spectrum have nonzero values, i.e., $\mathrm{BW}_{\text {eff }}\left(\frac{1}{\zeta T}\right) \triangleq \min \left\{\frac{1}{\zeta T}, 2 W\right\}$. Then, the DoF gain of the aFTN-NOMA scheme over the conventional NOMA scheme is defined by the ratio between the effective bandwidths of the two schemes, i.e.,

$$
\operatorname{Gain}_{\mathrm{DoF}} \triangleq \frac{\mathrm{BW}_{\text {eff }}\left(\frac{1}{\zeta T}\right)}{\mathrm{BW}_{\mathrm{eff}}\left(\frac{1}{T}\right)}=T \times \min \left\{\frac{1}{\zeta T}, 2 W\right\}
$$

Observe that with the reduction of $\zeta$, the SINR gain vanishes according to the properties of $\left|H_{\text {fo }}(f)\right|^{2}$ and $\left|H_{\text {tfo }}(f)\right|^{2}$, i.e., $\left|H_{\text {fo }}(f)\right|^{2}-\left|H_{\text {tfo }}(f)\right|^{2}$ is a non-increasing function in the range of $0 \leq \zeta \leq 1$. Furthermore, we can also observe that the DoF gain increases upon reducing $\zeta$. Therefore, we can see that there exists an intriguing trade-off between the SINR gain and DoF gain of the aFTN-NOMA schemes with respect to the symbol rate (compression factor).

Proposition 1 (Trade-off Between SINR Gain and DoF Gain): Upon increasing the symbol rate from $\zeta=1$ to $\zeta=1 /(1+\beta)$, the SINR gain of the aFTN-NOMA scheme decreases, while the DoF gain increases, and vice versa.

Particularly, it can be shown that the SINR gain achieves its maximum value when $\zeta=1$, i.e., for the aNOMA scheme, where there is no DoF gain. Furthermore, the DoF gain achieves its maximum when $\zeta \leq 1 /(1+\beta)$, where there is no SINR gain. Specifically, Definition 1 and Definition 2 have shown that both the folded-spectrum and the twisted foldedspectrum become exactly the same as the RRC spectrum within the frequency interval of $f \in\left[-\frac{1}{2 \zeta T}, \frac{1}{2 \zeta T}\right]$ for $\zeta \leq$ $1 /(1+\beta)$, in which case the upper- and lower-bounds in Theorem 3 are merged together and the corresponding maximum DoF gain is of value $2 W T$. Consequently, $R_{\mathbf{h}, \boldsymbol{\tau}, \zeta \leq 1 /(1+\beta)}^{k}$ of the aFTN-NOMA schemes associated with $\zeta \leq 1 /(1+\beta)$ is given by

$$
\begin{aligned}
& R_{\mathbf{h}, \boldsymbol{\tau}, \zeta \leq \frac{1}{1+\beta}}^{k} \\
&= \frac{1}{2 W} \int_{-W}^{W} \log _{2}\left(1+\frac{\left|h_{k}\right|^{2} P_{k}\left|H_{p}(f)\right|^{2}}{N_{0}+\sum_{l=k+1}^{K}\left|h_{l}\right|^{2} P_{l}\left|H_{p}(f)\right|^{2}}\right) \mathrm{d} f \\
& \text { bits } / \mathrm{s} / \mathrm{Hz} .
\end{aligned}
$$

Given (35), we can characterize the rate improvement of the aFTN-NOMA scheme over the conventional synchronous NOMA scheme in the high-SNR regime by the following corollary.

Corollary 3 (Achievable Rate Improvement of aFTN-NOMA Schemes): Given a sufficiently high SNR, the asymptotic instantaneous achievable rate of aFTN-NOMA schemes is $1+\beta$ times higher than that of the conventional synchronous NOMA scheme.

Proof: Considering the asymptotic instantaneous achievable rate of the conventional synchronous NOMA scheme given in (31), we have

$$
\begin{aligned}
\lim _{N_{0} \rightarrow 0} \frac{\frac{T}{1+\beta} \int_{-\frac{1+\beta}{2 T}}^{\frac{1+\beta}{2 T}} \log _{2}\left(1+\frac{\left|h_{k}\right|^{2} P_{k}\left|H_{p}(f)\right|^{2}}{N_{0}+\sum_{l=k+1}^{K}\left|h_{l}\right|^{2} P_{l}\left|H_{p}(f)\right|^{2}}\right) \mathrm{d} f}{\frac{1}{2 W T} \log _{2}\left(1+\frac{\left|h_{k}\right|^{2} P_{k} T}{N_{0}+\sum_{l=k+1}^{K}\left|h_{l}\right|^{2} P_{l} T}\right)} \\
=\frac{\log _{2}\left(1+\frac{\left|h_{k}\right|^{2} P_{k}}{\sum_{l=k+1}^{K}\left|h_{l}\right|^{2} P_{l}}\right)}{\frac{1}{2 W T} \log _{2}\left(1+\frac{\left|h_{k}\right|^{2} P_{k}}{\sum_{l=k+1}^{K}\left|h_{l}\right|^{2} P_{l}}\right)}=1+\beta .
\end{aligned}
$$

This completes the proof of Corollary 3 .

According to the conclusions from Theorem 3, Proposition 1 and Corollary 3, some important insights can be revealed for the family of aFTN-NOMA schemes.

- Similar to the aNOMA scheme, the achievable rate improvement of aFTN-NOMA schemes stems from the excess bandwidth of the signaling pulse, where both the upper-bound and the lower-bound in Theorem 3 are achievable according to Lemma 4 . Given a sufficiently high symbol rate, the upper- and lower-bounds in Theorem 3 are merged together, as suggested in (35).

- Proposition 1 has demonstrated the trade-off between the SINR gain and DoF gain. The physical interpretation of this trade-off is as follows. Given a higher symbol rate, the effect of spectral aliasing is mitigated, in which case the change of the folded-spectrum due to the phaserotation is limited. In particular, when $\zeta \leq 1 /(1+\beta)$, the spectral aliasing no longer exists and thus the SINR gain vanishes.

- Compared to the SINR gain, the DoF gain essentially leads to a higher achievable rate in the high-SNR regime, 
as shown in Corollary 3. In fact, the rate in (35) is essentially the ultimate achievable rate for a given signaling pulse and an SINR [20]. This is because the symbol rate is no lower than the bandwidth of the signaling pulse and therefore both the folded-spectrum and the twisted folded-spectrum become exactly the same as the underlying RRC spectrum, yielding the maximum capacity promised by the signaling pulse. However, for the sinc pulse, i.e., $\beta=0$, a higher symbol rate does not provide any improvement in terms of achievable rates, since the Nyquist symbol rate is simply twice the bandwidth of the signaling pulse, i.e., there is no spectral aliasing. In contrast to the aNOMA scheme and to the conventional synchronous NOMA scheme, the normalized achievable rate of the aFTN-NOMA scheme attained at a sufficiently high symbol rate only exhibits a constant gap to with respect to the $\beta=0$ case in the high-SNR regime. This is because the FTN transmission successes in fully exploiting the DoF promised by the siganling pulse [20].

\section{Implementations, Comparisons, and Extensions of the Con- sidered Schemes}

We have shown the advantages of aFTN-NOMA and aNOMA over the conventional synchronous NOMA scheme in terms of their achievable rate. However, it should be noted that both aFTN-NOMA and aNOMA suffer from ISI and therefore, they tend to impose an increased detection complexity [12]. A promising detector design could be based on the combination of both the MUI and ISI detections. In particular, the MUI can be efficiently dealt with by the SIC technique, given the channel disparities [3], while the ISI arising from both FTN and asynchronous transmissions can be mitigated by various mature detection methods conceived for ISI channels, e.g., [16], [35]. Although the related research on the detection issue is at its early stage, several potent detectors are available in the literature. We refer the interested readers to [12] and [36] for more details.

Note that our previous analysis in this section is purely based on baseband signaling, where we show that both aNOMA and aFTN-NOMA are superior to the conventional synchronous NOMA in terms of their achievable rates with arbitrarily given channel coefficients. Therefore, it is expected that both aFTN-NOMA and aNOMA will outperform conventional NOMA with any given distribution of channel coefficients. For a better understanding of aNOMA and aFTNNOMA, we briefly compare them to similar MA schemes as follows.

- aFTN-NOMA vs. OMA with FTN signaling: According to the previous analysis in this section, the achievable rate improvements attained by asynchronous transmission and FTN signaling accrue from the excess bandwidth of the signaling pulse, which is independent from the gain of NOMA gleaned from exploiting power discrepancies among different users. Furthermore, NOMA allows the information from different users to be transmitted in a time-sharing manner, which can be shown to have a higher achievable rate region than OMA [37]. Therefore,
aFTN-NOMA generally has a better performance than OMA with FTN signaling in terms of achievable rates, because OMA cannot exploit the power discrepancies and does not allow time-sharing among different users.

- aNOMA/aFTN-NOMA vs. RSMA: The success of RSMA lies in the rate-splitting, where the message of each user is divided into two parts, namely, a common part and a private part [38]. As the common part is supposed to be decoded by all the users, RSMA generally enjoys a reduced MUI compared to the conventional powerdomain NOMA [38]. Compared to RSMA, both aNOMA and aFTN-NOMA reduce the MUI by relying on asynchronous transmission, which is different from the principle of RSMA. However, it is generally not fair or practical to directly compare whether aNOMA/aFTN-NOMA or RSMA has inflicts lower MUI, because they both rely on the channel conditions, including the distributions of the channel coefficients and link delays. It is also worth mentioning that the advantages of both aNOMA and aFTN-NOMA become more pronounced for a higher excess bandwidth of the shaping pulse, while RSMA cannot make use of the excess bandwidth. An interesting discussion at this point may be the combination of asynchronous transmission and FTN signaling with RSMA. As RSMA and aNOMA/aFTN-NOMA enjoy advantages over NOMA from a range of different perspectives, their combination might lead to further rate improvements, which will be considered in our future work.

Now, we briefly discuss the potential extensions of the analysis in the previous subsections to more practical systems, including multi-carrier, multi-antenna, and multi-cell systems.

- Extension to multi-carrier systems: In multi-carrier systems, the signaling pulse is usually time-limited instead of being bandlimited. Thus, the schemes considered may be extended to multi-carrier systems by allowing asynchronous transmission and FTN signaling in the FD instead of the TD, which is essentially a type of spectrally efficient frequency domain multiplexing (FDM) signals [17], [18] with asynchronous transmissions. Our previous analysis could be extended to this case by interchanging the corresponding analysis between the FD and the TD.

- Extension to multi-antenna systems: Assume that the BS is equipped with multiple receive antennas, while the user has only a single transmit antenna. In this case, the BS may receive multiple copies of the transmitted signal (2) or (8) of each user at different link delays and channel coefficients. Naturally, SIC detection could be applied at the receiver for multiuser signal detection. The achievable rate analysis of such a system may also rely on the Toeplitz structure of the corresponding channel matrix after suitable combining of the signals received from different antennas.

- Extension to multi-cell systems: In multi-cell systems, the transmitted signals could be received by multiple cells. Then, depending on whether the cooperation between different cells is allowed, the inter-cell interference could 
be either exploited by cooperation or simply treated as noise for low-complexity processing. It should be noted that the signals transmitted by different users cannot be received by more than two BSs with perfect synchronization in practice, hence the considered asynchronous transmissions are permanently suitable for cooperative multi-cell systems. The achievable rate analysis of multicell systems may also rely on the analysis of singlecell settings. However, how the extra channel diversity gleaned from the potential multi-cell cooperations will improve the achievable rate may require further investigations.

Unfortunately, we have to leave the above interesting issues for our future works due to the page limitation.

\section{Numerical Results}

In this section, we compare the normalized achievable rates of the aNOMA scheme and the aFTN-NOMA scheme to that of the conventional synchronous NOMA scheme, where the actual achievable rate of the considered schemes are obtained based on (14) via the classic Monte Carlo method. In particular, we compare both the instantaneous rates and the ergodic rates relying on the randomly generated link delay for each user, where the link delay is assumed to follow a uniform distribution within the interval $[0,2 T]$ for each Monte Carlo realization. In order to verify our previous discussions, the instantaneous rates are calculated based on a typical NOMA transmission supporting $K=2$ and $K=3$ users, and the number of transmitted symbols for each user is set to $N=100$. On the other hand, the ergodic rates are calculated based on a typical single-cell settings from [3]. Without loss of generality, we adopt the equal power allocation for the different users for all the related simulations, i.e., $P_{k}=P$, for $k=1,2, \ldots K$.

\section{A. Normalized Instantaneous Achievable Rates for the Two- User Case}

We consider a specific channel realization, where the channel coefficients for the two users are given by $\left|h_{1}\right|^{2}=0.5$ and $\left|h_{2}\right|^{2}=0.5$ and we have $\frac{\left|h_{1}\right|^{2} P_{1}}{N_{0}}=\frac{\left|h_{2}\right|^{2} P_{2}}{N_{0}}=10 \mathrm{~dB}$. In order to obtain a general conclusion, we assume that each user has a random link delay. Given the channel coefficients, we adopt the Monte Carlo method to average the achievable rate with different link delays.

The achievable rate regions of the conventional synchronous NOMA, the aNOMA, and the aFTN-NOMA schemes are compared in Fig. 3, where the signaling pulse is the RRC pulse using $\beta=0.3$ and the compression factor for the aFTNNOMA scheme is $\zeta=0.75$. As shown in the figure, the conventional synchronous NOMA has the smallest achievable rate region among all the three schemes, while the aNOMA scheme only shows a marginal improvement. On the other hand, the aFTN-NOMA scheme can considerably improve the achievable rate as shown in Fig. 3, which is consistent with our previous analysis.

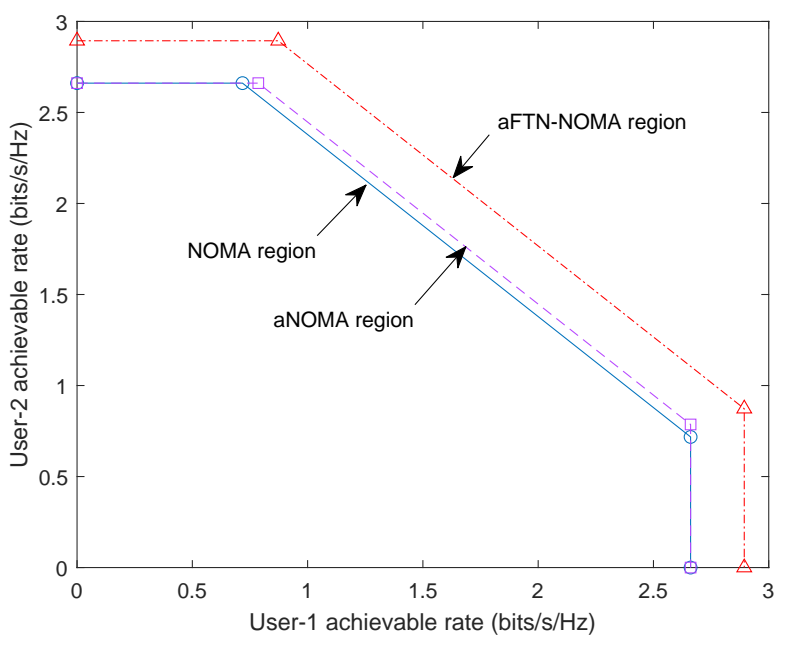

Fig. 3. The achievable rate regions of the conventional synchronous NOMA, the aNOMA, and the aFTN-NOMA schemes for $K=2$ users, where $\frac{\left|h_{1}\right|^{2} P_{1}}{N_{0}}=\frac{\left|h_{2}\right|^{2} P_{2}}{N_{0}}=10 \mathrm{~dB}$. The signaling pulse is the RRC pulse with $\beta=0.3$.

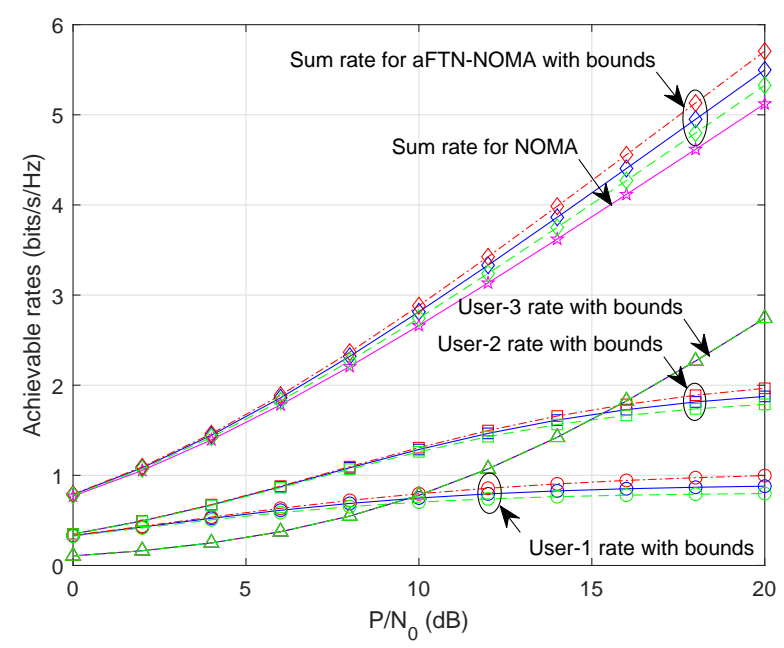

Fig. 4. The instantaneous achievable rate of aFTN-NOMA scheme for $\zeta=0.95$, where three users with equal power allocation are considered. The channel coefficients are given by $\left|h_{1}\right|^{2}=0.5,\left|h_{2}\right|^{2}=0.4$, and $\left|h_{3}\right|^{2}=0.1$, respectively. The red dash-dotted lines and the green dashed lines are the upper- and lower-bounds derived, while the blue solid line is the actual achievable rate. The signaling pulse is the RRC pulse with $\beta=0.3$.

\section{B. Normalized Instantaneous Achievable Rates for the Three- User Case}

Similar to the previous subsection, we consider a specific channel realization, where the channel coefficients of the three users are given by $\left|h_{1}\right|^{2}=0.5,\left|h_{2}\right|^{2}=0.4$, and $\left|h_{3}\right|^{2}=0.1$, respectively. We are interested in the achievable rate vs. the received SNR at the BS, which is defined by $\frac{\sum_{k=1}^{K}\left|h_{k}\right|^{2} P_{k}}{N_{0}}=$ $\frac{P}{N_{0}}$.

Fig. 4 shows the achievable rates of each user for the aFTN-NOMA scheme with $\zeta=0.95$, and $\beta=0.3$. As shown in the figure, the achievable rates of the aFTN-NOMA scheme are perfectly bounded by the upper- and lower-bounds derived. On the other hand, we observe that asynchronous FTN 


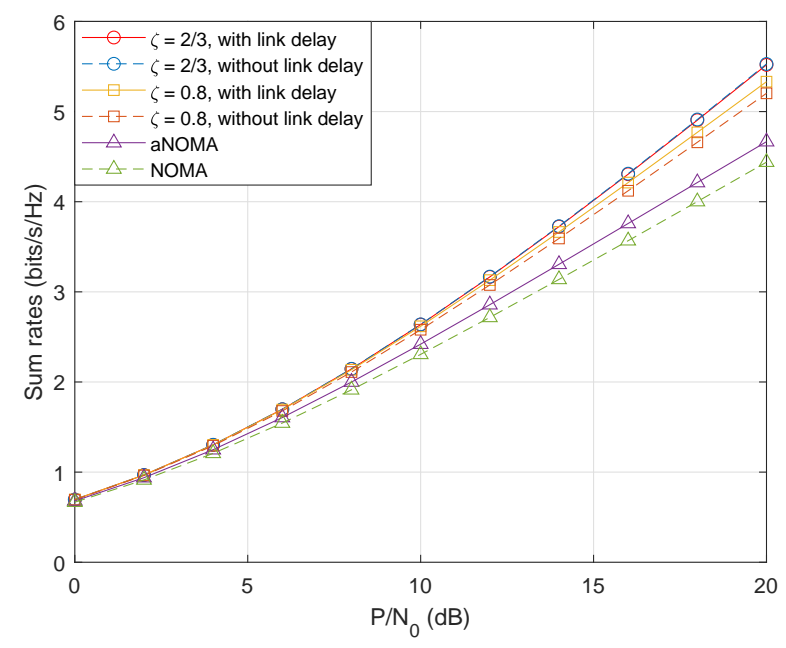

Fig. 5. An illustration of the trade-off between the SINR gain and the DoF gain for aFTN-NOMA schemes with different values of $\zeta$, where three users with equal power allocation are considered. The channel coefficients are given by $\left|h_{1}\right|^{2}=0.5,\left|h_{2}\right|^{2}=0.4$, and $\left|h_{3}\right|^{2}=0.1$, respectively. The signaling pulse is the RRC pulse with $\beta=0.5$.

transmission indeed attains rate improvements. Specifically, there is a $1.3 \mathrm{~dB}$ gain for the aFTN-NOMA scheme with $\zeta=0.95$ compared to the conventional synchronous NOMA scheme at a rate of $5 \mathrm{bits} / \mathrm{s} / \mathrm{Hz}$.

Let us demonstrate the SINR vs. DoF trade-off with $\beta=$ 0.5 in Fig. 5, where we show the sum-rates of the aFTNNOMA scheme, the aNOMA scheme, and the conventional synchronous NOMA scheme. For comparison, we also provide the corresponding synchronous rates without link delays. Specifically, we consider three cases at different symbol rates, i.e., $\zeta=1, \zeta=0.8$, and $\zeta=2 / 3$, respectively. As indicated in Proposition 1, the SINR gain reduces with the reduction of $\zeta$ from $\zeta=1$ to $\zeta=\frac{1}{1+\beta}=\frac{2}{3}$, while the DoF gain increases. As observed from the figure, the SINR gain indeed decreases (corresponding to the shift along the $\mathrm{x}$-axis) for a smaller $\zeta$, while the DoF gain (corresponding to the slope of the curves) increases. In particular, we notice that with $\zeta=2 / 3$, the curves of the aFTN-NOMA schemes operating with or without link delay are almost overlapped, which indicates that the SINR gain due to the link delay vanishes in this case. The observations confirm the accuracy of our derivations in Section III.

\section{Normalized Ergodic Achievable Rates for Single-Cell Se- tups}

In this subsection, we focus our attention on the ergodic achievable rates of both aFTN-NOMA and aNOMA, and compare them to that of the conventional power-domain NOMA in a single-cell system. In particular, we adopt the system settings from [3] for our simulations, where we assume that the cell is modeled by a pair of concentric ring-shaped discs. The inner radius $D_{0}$ is introduced to model the minimum propagation path loss [3], while the outer radius $D_{1}$ represents the cell size. Furthermore, we assume that the BS is located at the center of the cell and all the users are uniformly scattered
TABLE II

RElated PARAMETERS FOR Simulations

\begin{tabular}{|l|r|}
\hline Packet length $N$ & 100 \\
\hline Nyquist symbol period $T$ & 1 \\
\hline Maximum value of link delay & $2 T$ \\
\hline Inner cell radius $D_{0}$ & $50 \mathrm{~m}$ \\
\hline Outer cell radius $D_{1}$ & {$[75,100,200,300,400,500] \mathrm{m}$} \\
\hline Number of users & {$[2,4,8,16,32,64,128]$} \\
\hline Path loss exponent $\alpha$ & 3.76 \\
\hline Noise PSD $N_{0}$ & $-80 \mathrm{dBm}$ \\
\hline
\end{tabular}

within the two concentric ring-shaped discs. To characterize the system's SNR, we adopt the definitions from [3], where the total average received SNR of all the users at the BS is defined by

$$
\mathrm{SNR}_{\mathrm{sum}} \triangleq \frac{P_{\max }}{N_{0}} \overline{|h|^{2}} .
$$

In (37), $\overline{|h|^{2}}$ denotes the average channel power gain with respect to the cell size and path loss model and it is calculated based on Equation (12) of [3]. The term $P_{\max }$ in (37) denotes the total transmit power of the BS, which is adjusted adaptively for different cell sizes to provide the required $\mathrm{SNR}_{\text {sum }}$. Meanwhile, the noise PSD in (37) is set to be $N_{0}=-80$ $\mathrm{dBm}$. For reference, we summarize the related parameters in Table II.

We show the ergodic sum-rates of both aFTN-NOMA and aNOMA in comparison to that of NOMA in Fig. 6(a) versus $\mathrm{SNR}_{\text {sum }}$ and the numbers of users, where $\beta=0.3, \tau=0.75$, and $D_{1}=75$. As observed from Fig. 6(a), the ergodic sumrates of both aFTN-NOMA and aNOMA are higher than the synchronous NOMA benchmark for various system SNRs, where the sum-rate improvements increase with $\mathrm{SNR}_{\text {sum. }}$. Indeed, both the DoF and the MUI are dominant factors in determining the achievable rates in the high-SNR regime. Therefore, FTN signaling and asynchronous transmission lead to beneficial rate improvements due to the exploitations of DoF and the mitigation of MUI. On the other hand, we also observe from Fig. 6(a) that aNOMA achieves a higher sumrate improvement for more users, while the sum-rate gap between aFTN-NOMA and NOMA is relatively constant for more than 8 users. This is because the rate improvement of aNOMA arises from the MUI mitigation, while the rate improvement of aFTN-NOMA comes from its DoF gain. As the MUI increases with more users in the cell, the MUI mitigation leads to an increased rate improvement. However, as the DoF gain of aFTN-NOMA comes from the excess bandwidth of the signaling pulse, which does not change with the number of users in the cell, the rate improvement remains relatively constant for different number of users.

We portray the performance comparisons among NOMA, aNOMA, and aFTN-NOMA in terms of the cell size in Fig. 6(b), where $\beta=0.3, \tau=0.75, K=128$, and $\mathrm{SNR}_{\text {sum }}=20 \mathrm{~dB}$. As indicated in Fig. 6(b), the sum-rate gap between aFTN-NOMA and NOMA is relatively constant, while the sum-rate improvement of aNOMA is reduced for large cells. This observation is not unexpected because the DoF gain of aFTN-NOMA does not change with the channel characteristics. However, with the transmitted power fixed, the 


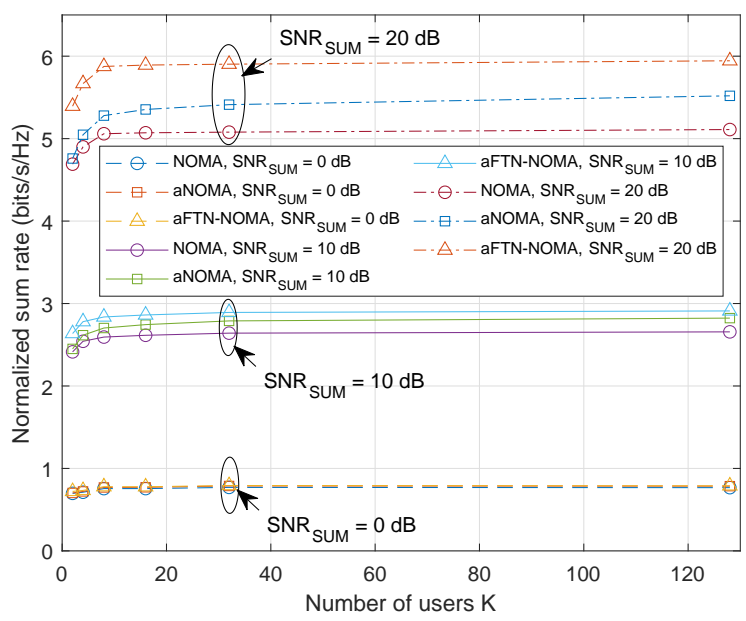

(a) Normalized sum-rate vs. number of users with different SNRs.

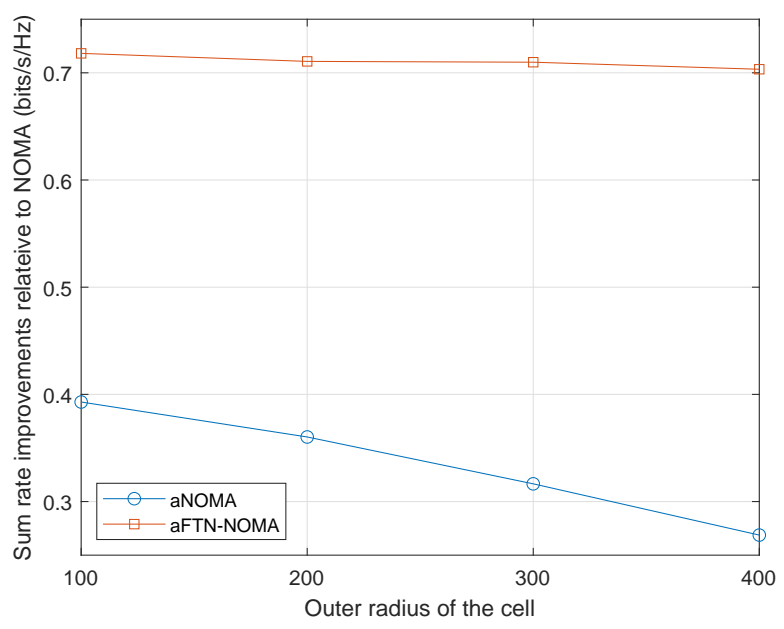

(b) Normalized sum-rate vs. cell range.

Fig. 6. Ergodic sum-rate analysis for the considered schemes, where the related simulation parameters are given in Table II.

MUI generally reduces for larger cells and therefore, the rate improvement of aNOMA also reduces.

The sum-rate performances versus roll-off factors are demonstrated in Fig. 7 (a), where $K=16, D_{1}=75$, and $\mathrm{SNR}_{\text {sum }}=40 \mathrm{~dB}$. In particular, we set $\tau=0.5$ for aFTNNOMA system as it is sufficient to obtain the full DoF gains for $\beta \in[0,1]$. We observe that the sum-rate of all three schemes decreases for a larger $\beta$ due to the bandwidth normalization. However, the sum-rates of both NOMA and aNOMA reduce significantly compared to that of the aFTNNOMA. This is because aFTN-NOMA efficiently exploits the DoF gain based on the excess bandwidth, which is consistent with our analysis in Section III-B. On the other hand, the sum-rate gap between NOMA and aNOMA increases for a larger roll-off factor. This is due to the fact that a larger excess bandwidth offers a stronger interference mitigation capability for aNOMA, which is also consistent with our discussions in Section III-A.

The comparison of complementary cumulative distribution

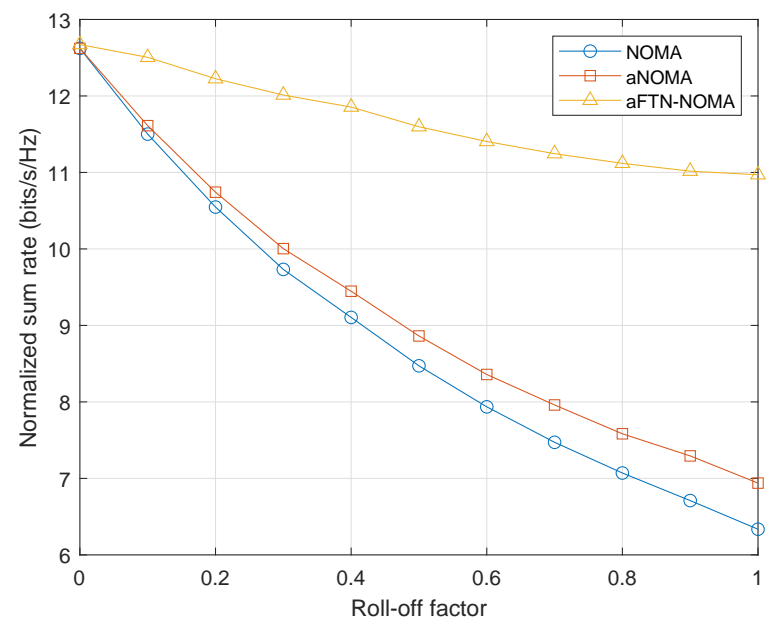

(a) Sum rate vs. roll-off factors.

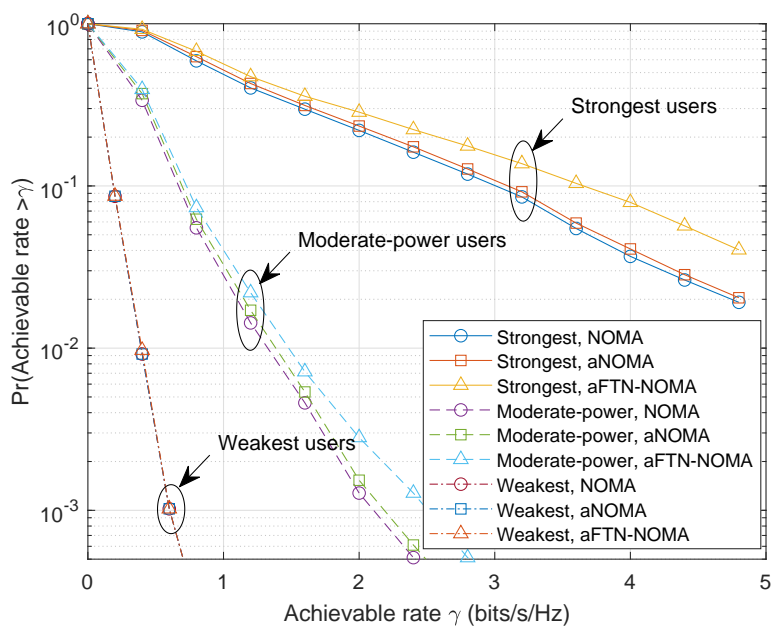

(b) CCDFs for different schemes.

Fig. 7. Achievable rate analysis for the considered schemes, where the related simulation parameters are given in Table II.

functions (CCDFs) of different users is given in Fig. 7(b), where $K=8, \beta=0.3, \tau=0.75, D_{1}=500$, and $\mathrm{SNR}_{\text {sum }}=20 \mathrm{~dB}$. Specifically, we show the CCDFs of the strongest user, a moderate-power user, and the weakest user in the schemes considered. As indicated from the figure, the CCDFs of both aNOMA and aFTN-NOMA show better performance than that of NOMA for both the strongest user and moderate-power user. However, the CCDFs of the weakest users are almost the same for the three schemes. This is because the weakest user generally has low received SNR while the MUI is eliminated thanks to the SIC detection. In this case, neither the MUI mitigation, nor the DoF gain may be able to offer large rate improvements. Consequently, the performances of aFTN-NOMA, aNOMA, and NOMA are similar.

\section{CONCLUSIONS}

In this paper, we investigated the aNOMA and aFTNNOMA schemes with the objective of improving the achiev- 


$$
\begin{aligned}
& I_{\mathbf{h}, \boldsymbol{\tau}, \zeta}\left(\mathbf{y}_{k} ; \mathbf{x}_{k} \mid \mathbf{x}_{1}, \ldots, \mathbf{x}_{k-1}\right) \\
= & \frac{1}{2} \log _{2} \operatorname{det}\left[\left(\sum_{l=k}^{K}\left|h_{l}\right|^{2} E_{s}[l] \tilde{\mathbf{G}}_{l, k} \tilde{\mathbf{G}}_{l, k}^{\mathrm{T}}+N_{0} \tilde{\mathbf{G}}_{k, k}\right)\left(\sum_{l=k+1}^{K}\left|h_{l}\right|^{2} E_{s}[l] \tilde{\mathbf{G}}_{l, k} \tilde{\mathbf{G}}_{l, k}^{\mathrm{T}}+N_{0} \tilde{\mathbf{G}}_{k, k}\right)^{-1}\right] \\
= & \frac{1}{2} \log _{2} \operatorname{det}\left[\mathbf{I}_{N \times N}+\frac{\left|h_{k}\right|^{2} E_{s}[k] \tilde{\mathbf{G}}_{k, k} \tilde{\mathbf{G}}_{k, k}^{\mathrm{T}}}{N_{0}}\left(\tilde{\mathbf{G}}_{k, k}+\frac{\sum_{l=k+1}^{K}\left|h_{l}\right|^{2} E_{s}[l]}{N_{0}} \tilde{\mathbf{G}}_{l, k} \tilde{\mathbf{G}}_{l, k}^{\mathrm{T}}\right)^{-1}\right] .
\end{aligned}
$$

able rate of conventional NOMA transmissions. Specifically, we derived the corresponding achievable rate upper- and lower-bounds by invoking Szegö's Theorem. We showed that asynchronous transmissions result in an SINR gain, while increasing the symbol rate may potentially lead to a DoF gain. More importantly, we unveiled that the associated SINR vs. DoF gain trade-off. In particular, we also showed that the SINR or DoF gains are related to the potential spectral aliasing and discussed the connections between the effect of spectral aliasing and the link delay as well as the symbol rate. Our simulation results agreed with our analysis and demonstrated a significant achievable rate gain compared to the conventional NOMA transmission.

\section{APPENDIX A}

\section{PROOF OF LEMMA 1}

According to the chain rule and the calculation of the entropy of a multivariate normal distribution [37], we have

$$
\begin{aligned}
& I_{\mathbf{h}, \boldsymbol{\tau}, \zeta}\left(\mathbf{y}_{k} ; \mathbf{x}_{k} \mid \mathbf{x}_{1}, \ldots, \mathbf{x}_{k-1}\right) \\
= & h_{\mathbf{h}, \boldsymbol{\tau}, \zeta}\left(\mathbf{y}_{k} \mid \mathbf{x}_{1}, \ldots, \mathbf{x}_{k-1}\right)-h_{\mathbf{h}, \boldsymbol{\tau}, \zeta}\left(\mathbf{y}_{k} \mid \mathbf{x}_{1}, \ldots, \mathbf{x}_{k}\right) \\
= & \frac{1}{2} \log _{2}(2 \pi e)^{N} \operatorname{det}\left(\sum_{l=k}^{K}\left|h_{l}\right|^{2} E_{s}[l] \tilde{\mathbf{G}}_{l, k} \tilde{\mathbf{G}}_{l, k}^{\mathrm{T}}+N_{0} \tilde{\mathbf{G}}_{k, k}\right) \\
& -\frac{1}{2} \log _{2}(2 \pi e)^{N} \operatorname{det}\left(\sum_{l=k+1}^{K}\left|h_{l}\right|^{2} E_{s}[l] \tilde{\mathbf{G}}_{l, k} \tilde{\mathbf{G}}_{l, k}^{\mathrm{T}}+N_{0} \tilde{\mathbf{G}}_{k, k}\right) .
\end{aligned}
$$

Furthermore, according to the property of the matrix determinant, we have (39) as shown at the top of this page. This completes the proof of Lemma 1.

\section{APPENDIX B}

\section{PROOF OF LEMMA 3}

According to the definition of $\mathbf{T}_{l, k}$, to verify the positive definiteness of $\mathbf{T}_{l, k}$ is equivalent to verify that $\tilde{\mathbf{G}}_{l, k}$ has a positive determinant. It can be observed from (12) that $\tilde{\mathbf{G}}_{l, k}$ is a Gram matrix of nonzero energy functions $p(t+n \zeta T+\tau[l]-\tau[k])$, for $n=0, \ldots, N-1$, where the element of the $i$-th row and $j$-th column is given by the inner product of $p(t+i \zeta T+\tau[l]-\tau[k])$ and $p(t+j \zeta T+\tau[l]-\tau[k])$. Note that the determinant of $\tilde{\mathbf{G}}_{l, k}$ is non-negative [39]. Therefore, we only have to verify that $\tilde{\mathbf{G}}_{l, k}$ has a nonzero determinant. Gram's criterion [39] indicates that for a set of strictly bandlimited functions $p(t+n \zeta T+\tau[l]-\tau[k])$ having a finite energy in their frequency interval, the corresponding Gram matrix $\tilde{\mathbf{G}}_{l, k}$ has a nonzero determinant if and only if the set of functions $p(t+n \zeta T+\tau[l]-\tau[k])$, for $n=0, \ldots, N-1$ are linearly independent. In order to prove the linear independence, we consider Proposition 5.1.1 of [40], which indicates that a sufficient condition for the function set to be linearly independent is that

$$
\lim _{n \rightarrow \infty} \tilde{g}[n, \tau[l]-\tau[k]]=0,
$$

for any $1 \leq l, k \leq K[33]$. Upon recalling (10), to prove (40), we have to verify

$$
\lim _{n \rightarrow \infty} \int_{-\infty}^{\infty}\left|H_{p}(f)\right|^{2} \exp (j 2 \pi f(n \zeta T+\tau[l]-\tau[k])) \mathrm{d} f=0 .
$$

Note that $\tau[l]-\tau[k]$ is constant for any given $l$ and $k$. Therefore, (41) holds due to the Riemann-Lebesgue Lemma [41]. Furthermore, since $\tilde{\mathbf{G}}_{l, k}$ is a Toeplitz matrix, $\mathbf{T}_{l, k}$ is asymptotically a Toeplitz matrix, due to the fact that the product of two Toeplitz matrices is also asymptotically Toeplitz [22]. On the other hand, it can be shown that the summation of two Toeplitz matrices is also a Toeplitz matrix, while the inverse of a Toeplitz matrix is asymptotically a Toeplitz matrix [22]. Therefore, it can be shown that $\mathbf{P}_{k}$ is asymptotically a Toeplitz matrix. Meanwhile, we have verified that $\tilde{\mathbf{G}}_{l, k}$ is a positive definite Toeplitz matrix. Then, it can be shown that $\mathbf{P}_{k}$ is also positive definite, because the product of positive definite matrices is also positive definite. This completes the proof of Lemma 3.

\section{APPENDIX C \\ PROOF OF LEMMA 4}

As we know that the spectrum of RRC pulse is $\left|H_{p}(f)\right|^{2}$ strictly non-negative, we have

$$
\begin{aligned}
& \sum_{k=-\infty}^{\infty}\left|H_{p}\left(f-\frac{k}{\zeta T}\right)\right|^{2} e^{-j 2 \pi \gamma k} \\
\leq & \sum_{k=-\infty}^{\infty}\left|H_{p}\left(f-\frac{k}{\zeta T}\right)\right|^{2}\left|e^{-j 2 \pi \gamma k}\right|=\left|H_{\text {fo }}(f)\right|^{2},
\end{aligned}
$$

where the bound becomes exact if $\gamma=0$. On the other hand, notice that $\left|H_{p}(f)\right|^{2}$ is strictly bandlimited within the 
frequency interval $|f| \leq \frac{1+\beta}{2 T}$. Thus, for $f \in\left[-\frac{1}{2 \zeta T}, \frac{1}{2 \zeta T}\right]$, we have

$$
\begin{aligned}
& \sum_{k=-\infty}^{\infty}\left|H_{p}\left(f-\frac{k}{\zeta T}\right)\right|^{2} e^{-j 2 \pi \gamma k} \\
= & \left|H_{p}(f)\right|^{2}+\left|H_{p}\left(f-\frac{1}{\zeta T}\right)\right|^{2} e^{-j 2 \pi \gamma}+\left|H_{p}\left(f+\frac{1}{\zeta T}\right)\right|^{2} e^{j 2 \pi \gamma} \\
\geq & \left|H_{p}(f)\right|^{2}-\left|H_{p}\left(f-\frac{1}{\zeta T}\right)\right|^{2}-\left|H_{p}\left(f+\frac{1}{\zeta T}\right)\right|^{2} \\
= & \left|H_{\mathrm{tfo}}(f)\right|^{2}
\end{aligned}
$$

Next, we discuss the achievability of the derived bounds. For $\beta=0$, it is not hard to notice that both $\left|H_{\mathrm{tfo}}(f)\right|^{2}$ and $\left|H_{\text {fo }}(f)\right|^{2}$ become the same as the sinc spectrum $\left|H_{\text {sinc }}(f)\right|^{2}$. In this case, both the upper-bound of (42) and the lower-bound of (43) are achieved. Furthermore, we note that both $\left|H_{\text {fo }}(f)\right|^{2}$ and $\left|H_{\mathrm{tfo}}(f)\right|^{2}$ become the exact RRC spectrum $\left|H_{p}(f)\right|^{2}$, when $\zeta \leq 1 /(1+\beta)$. Thus, both the upper-bound of (42) and the lower-bound of (43) are also achieved in this case. This completes the proof of Lemma 4.

\section{APPENDIX D \\ PROOF OF THEOREM 1}

Upon recalling (10), we have

$$
\begin{aligned}
\tilde{G}_{l, k}(2 \pi f \zeta T) & =\sum_{n=-\infty}^{\infty} \tilde{g}_{\zeta}[n, \Delta \tau] e^{-j 2 \pi n \zeta T f} \\
& =\int_{-\infty}^{\infty}\left|H_{p}(\lambda)\right|^{2} e^{j 2 \pi \lambda \Delta \tau} \sum_{n=-\infty}^{\infty} e^{j 2 \pi n \zeta T(\lambda-f)} \mathrm{d} \lambda .
\end{aligned}
$$

Substituting the Poisson summation formula, i.e., $\sum_{k=-\infty}^{\infty} e^{j 2 \pi k x}=\sum_{k=-\infty}^{\infty} \delta(x+k)$, into (44) yields

$$
\begin{aligned}
& \tilde{G}_{l, k}(2 \pi f \zeta T) \\
= & \int_{-\infty}^{\infty}\left|H_{p}(\lambda)\right|^{2} e^{j 2 \pi \lambda \Delta \tau} \sum_{n=-\infty}^{\infty} \delta(\zeta T(\lambda-f)+n) \mathrm{d} \lambda \\
= & \frac{1}{\zeta T} \int_{-\infty}^{\infty}\left|H_{p}(\lambda)\right|^{2} e^{j 2 \pi \lambda \Delta \tau} \sum_{n=-\infty}^{\infty} \delta\left(\lambda-\left(f-\frac{n}{\zeta T}\right)\right) \mathrm{d} \lambda \\
= & \frac{1}{\zeta T} \sum_{n=-\infty}^{\infty}\left|H_{p}\left(f-\frac{n}{\zeta T}\right)\right|^{2} e^{j 2 \pi\left(f-\frac{n}{\zeta T}\right) \Delta \tau},
\end{aligned}
$$

where (45) is due to the property of the Dirac delta function. Therefore, by considering Lemma 4, (46) can be bounded by

$$
\frac{1}{\zeta T}\left|H_{\mathrm{tfo}}(f)\right|^{2} \leq \tilde{G}_{l, k}(2 \pi f \zeta T) \leq \frac{1}{\zeta T}\left|H_{\mathrm{fo}}(f)\right|^{2} .
$$

It should be noted that the bounds derived are based on the conclusions from Lemma 4. Therefore, those bounds become exact when the conditions stated in Lemma 4 are satisfied. This completes the proof of Theorem 1 .

\section{APPENDIX E}

PROOF OF THEOREM 2

Similar to the proof of Theorem 1, we substitute (10) into (21) and obtain

$$
\begin{aligned}
& \tilde{T}_{l, k}(2 \pi f \zeta T) \\
= & \sum_{n=-\infty}^{\infty} \sum_{m=-\infty}^{\infty} \tilde{g}_{\zeta}[m, \Delta \tau] \tilde{g}_{\zeta}[m-n, \Delta \tau] e^{-j 2 \pi n \zeta T f} \\
= & \sum_{m=-\infty}^{\infty} \tilde{g}_{\zeta}[m, \Delta \tau] \int_{-\infty}^{\infty}\left|H_{p}(\lambda)\right|^{2} e^{j 2 \pi \lambda(m \zeta T+\Delta \tau)} \\
& \sum_{n=-\infty}^{\infty} e^{-j 2 \pi n \zeta T(\lambda+f)} \mathrm{d} \lambda .
\end{aligned}
$$

Upon considering the Poisson summation formula, we have

$$
\begin{aligned}
& \tilde{T}_{l, k}(2 \pi f \zeta T) \\
= & \sum_{m=-\infty}^{\infty} \tilde{g}_{\zeta}[m, \Delta \tau] \int_{-\infty}^{\infty}\left|H_{p}(\lambda)\right|^{2} e^{j 2 \pi \lambda(m \zeta T+\Delta \tau)} \\
& \sum_{n=-\infty}^{\infty} \delta(n-\zeta T(\lambda+f)) \mathrm{d} \lambda \\
= & \frac{1}{\zeta T} \sum_{m=-\infty}^{\infty} \tilde{g}_{\zeta}[m, \Delta \tau] e^{j 2 \pi f(m \zeta T+\Delta \tau)} \\
& \sum_{n=-\infty}^{\infty}\left|H_{p}\left(f-\frac{n}{\zeta T}\right)\right|^{2} e^{-j 2 \pi m n-\frac{n \Delta \tau}{\zeta T}} .
\end{aligned}
$$

Upon recalling Lemma 4, (49) can be upper-bounded by

$$
\tilde{T}_{l, k}(2 \pi f \zeta T) \leq \frac{1}{\zeta T}\left|H_{\text {fo }}(f)\right|^{2} \sum_{m=-\infty}^{\infty} \tilde{g}_{\zeta}[m, \Delta \tau] e^{j 2 \pi f(m \zeta T+\Delta \tau)} .
$$

Moreover, by considering Theorem 1, (50) can be further upper-bounded by

$$
\tilde{T}_{l, k}(2 \pi f \zeta T) \leq\left(\frac{1}{\zeta T}\left|H_{\text {fo }}(f)\right|^{2}\right)^{2} .
$$

On the other hand, (49) can be lower-bounded according to Lemma 4 by

$\tilde{T}_{l, k}(2 \pi f \zeta T) \geq \frac{1}{\zeta T}\left|H_{\mathrm{tfo}}(f)\right|^{2} \sum_{m=-\infty}^{\infty} \tilde{g}_{\zeta}[m, \Delta \tau] e^{j 2 \pi f(m \zeta T+\Delta \tau)}$.

Again, by considering Theorem 1, (50) can be further lowerbounded by

$$
\tilde{T}_{l, k}(2 \pi f \zeta T) \geq\left(\frac{1}{\zeta T}\left|H_{\mathrm{tfo}}(f)\right|^{2}\right)^{2} .
$$

It should be noted that the bounds derived are based on the conclusions from Lemma 4. Therefore, those bounds become exact when the conditions stated in Lemma 4 are met. This completes the proof of Theorem 2 . 


$$
\begin{aligned}
R_{\mathbf{h}, \boldsymbol{\tau}, \zeta}^{k} & \triangleq \lim _{N \rightarrow \infty} \frac{1}{N} I_{\mathbf{h}, \tau, \zeta=1}\left(\mathbf{y}_{k} ; \mathbf{x}_{k} \mid \mathbf{x}_{1}, \ldots, \mathbf{x}_{k-1}\right) \\
& \left.=\frac{1}{4 \pi} \int_{-\pi}^{\pi} \log _{2}\left(1+\frac{\left|h_{k}\right|^{2} E_{s}[k] \tilde{T}_{k, k}(\omega)}{N_{0}}\left(\tilde{G}_{k, k}(\omega)+\frac{\sum_{l=k+1}^{K}\left|h_{l}\right|^{2} E_{s}[l] \tilde{T}_{l, k}(\omega)}{N_{0}}\right)\right)^{-1}\right) \mathrm{d} \omega \\
& =\frac{\zeta T}{2} \int_{-\frac{1}{2 \zeta T}}^{\frac{1}{2 \zeta T}} \log _{2}\left(1+\frac{\left|h_{k}\right|^{2} E_{s}[k] \tilde{T}_{k, k}(2 \pi \zeta T f)}{N_{0} \tilde{G}_{k, k}(2 \pi \zeta T f)+\sum_{l=k+1}^{K}\left|h_{l}\right|^{2} E_{s}[l] \tilde{T}_{l, k}(2 \pi \zeta T f)}\right) \mathrm{d} f .
\end{aligned}
$$

$$
\begin{aligned}
R_{\mathbf{h}, \boldsymbol{\tau}, \zeta}^{k} & \geq \frac{\zeta T}{2} \int_{-\frac{1}{2 \zeta T}}^{\frac{1}{2 \zeta T}} \log _{2}\left(1+\frac{\left|h_{k}\right|^{2} E_{s}[k]\left(\frac{\left|H_{\mathrm{fo}}(f)\right|^{2}}{\zeta T}\right)^{2}}{N_{0} \frac{\left|H_{\mathrm{fo}}(f)\right|^{2}}{\zeta T}+\sum_{l=k+1}^{K}\left|h_{l}\right|^{2} E_{s}[l]\left(\frac{\left|H_{\mathrm{fo}}(f)\right|^{2}}{\zeta T}\right)^{2}}\right) \mathrm{d} f \\
& =\frac{\zeta T}{2} \int_{-\frac{1}{2 \zeta T}}^{\frac{1}{2 \zeta T}} \log _{2}\left(1+\frac{\left|h_{k}\right|^{2} P_{k}\left|H_{\mathrm{fo}}(f)\right|^{2}}{N_{0}+\sum_{l=k+1}^{K}\left|h_{l}\right|^{2} P_{l}\left|H_{\mathrm{fo}}(f)\right|^{2}}\right) \mathrm{d} f .
\end{aligned}
$$

\section{APPENDIX F}

PROOF OF THEOREM 3

We apply Szegö's Theorem to (14), yielding (54) as shown at the top this page. Note that the integral range in (54) is within the frequency interval $f \in\left[-\frac{1}{2 \zeta T}, \frac{1}{2 \zeta T}\right]$. Therefore, the bounds in Theorem 1 and Theorem 2 can be applied for analysis. By observing that $\tilde{G}_{k, k}(2 \pi \zeta T f)=\frac{1}{\zeta T}\left|H_{\mathrm{fo}}(f)\right|^{2}$ and $\tilde{T}_{k, k}(2 \pi \zeta T f)=\left(\frac{1}{\zeta T}\left|H_{\text {fo }}(f)\right|^{2}\right)^{2}$, we have

$$
\begin{aligned}
& R_{\mathbf{h}, \boldsymbol{\tau}, \zeta}^{k} \\
\leq & \frac{\zeta T}{2} \int_{-\frac{1}{2 \zeta T}}^{\frac{1}{2 \zeta T}} \log _{2}\left(1+\frac{\left|h_{k}\right|^{2} E_{s}[k]\left(\frac{\left|H_{\mathrm{fo}}(f)\right|^{2}}{\zeta T}\right)^{2}}{\frac{N_{0}\left|H_{\mathrm{fo}}(f)\right|^{2}}{\zeta T}+\sum_{l=k+1}^{K}\left|h_{l}\right|^{2} E_{s}[l]\left(\frac{\left|H_{\mathrm{tfo}}(f)\right|^{2}}{\zeta T}\right)^{2}}\right) \mathrm{d} f,
\end{aligned}
$$

which can be further simplified as

$$
\begin{aligned}
& R_{\mathbf{h}, \boldsymbol{\tau}, \zeta}^{k} \\
\leq & \frac{\zeta T}{2} \int_{-\frac{1}{2 \zeta T}}^{\frac{1}{2 \zeta T}} \log _{2}\left(1+\frac{\left|h_{k}\right|^{2} P_{k}\left|H_{\mathrm{fo}}(f)\right|^{2}}{N_{0}+\sum_{l=k+1}^{K}\left|h_{l}\right|^{2} P_{l}\left|H_{\mathrm{tfo}}(f)\right|^{2} \rho(f)}\right) \mathrm{d} f .
\end{aligned}
$$

On the other hand, we obtain (57) as shown at the top of this page. Furthermore, by considering the symbol rate $\frac{1}{\zeta T}$, the signal dimension, and the signal bandwidth $2 W$, the bounds in (56) and (57) can be normalized as shown in (29) and (30). Finally, it can be shown that the bounds derived become exact when the conditions stated in Lemma 4 are met. This completes the proof of Theorem 3.

\section{REFERENCES}

[1] S. Li, Z. Wei, W. Yuan, J. Yuan, B. Bai, and D. W. K. Ng, "On the achievable rates of uplink NOMA with asynchronized transmission," in Proc. IEEE Wireless Commun. Net. Conf., 2020, pp. 1-6.

[2] Z. Ding, X. Lei, G. K. Karagiannidis, R. Schober, J. Yuan, and V. K. Bhargava, "A survey on non-orthogonal multiple access for 5G networks: Research challenges and future trends," IEEE J. Sel. Areas Commun., vol. 35, no. 10, pp. 2181-2195, Oct. 2017.

[3] Z. Wei, L. Yang, D. W. K. Ng, J. Yuan, and L. Hanzo, "On the performance gain of NOMA over OMA in uplink communication systems," IEEE Trans. Commun., vol. 68, no. 1, pp. 536-568, Jan. 2020.

[4] Q. Wu and R. Zhang, "Towards smart and reconfigurable environment: Intelligent reflecting surface aided wireless network," IEEE Commun. Mag., vol. 58, no. 1, pp. 106-112, Nov. 2020.

[5] Y. Wang, J. Wang, D. W. K. Ng, R. Schober, and X. Gao, "A minimum error probability NOMA design," IEEE Trans. Wireless Commun., Jul. 2021.

[6] B. Rimoldi and R. Urbanke, "A rate-splitting approach to the Gaussian multiple-access channel," IEEE Trans. Inf. Theory, vol. 42, no. 2, pp. 364-375, Mar. 1996.

[7] B. Clerckx, H. Joudeh, C. Hao, M. Dai, and B. Rassouli, "Rate splitting for MIMO wireless networks: A promising PHY-layer strategy for LTE evolution," IEEE Commun. Mag., vol. 54, no. 5, pp. 98-105, May 2016.

[8] Z. Ding and H. Vincent Poor, "A simple design of IRS-NOMA transmission," IEEE Commun. Lett., vol. 24, no. 5, pp. 1119-1123, Feb. 2020.

[9] M. Moltafet, N. M. Yamchi, M. R. Javan, and P. Azmi, "Comparison study between PD-NOMA and SCMA," IEEE Trans. Veh. Technol., vol. 67, no. 2, pp. 1830-1834, Feb. 2018.

[10] H. Haci, H. Zhu, and J. Wang, "Performance of non-orthogonal multiple access with a novel asynchronous interference cancellation technique," IEEE Trans. Commun., vol. 65, no. 3, pp. 1319-1335, Mar. 2017.

[11] X. Zou, B. He, and H. Jafarkhani, "An analysis of two-user uplink asynchronous non-orthogonal multiple access systems," IEEE Trans. Wireless Commun., vol. 18, no. 2, pp. 1404-1418, Jan. 2019.

[12] W. Yuan, N. Wu, Q. Guo, D. W. K. Ng, J. Yuan, and L. Hanzo, "Iterative joint channel estimation, user activity tracking, and data detection for FTN-NOMA systems supporting random access," IEEE Trans. Commun., vol. 68, no. 5, pp. 2963-2977, May 2020. 
[13] J. B. Anderson, F. Rusek, and V. Öwall, "Faster-than-Nyquist signaling," Proc. IEEE, vol. 101, no. 8, pp. 1817-1830, Aug. 2013.

[14] S. Li, J. Yuan, B. Bai, and N. Benvenuto, "Code-based channel shortening for faster-than-Nyquist signaling: Reduced-complexity detection and code design," IEEE Trans. Commun., vol. 68, no. 7, pp. 3996-4011, Jul. 2020.

[15] S. Sugiura and L. Hanzo, "Frequency-domain-equalization-aided iterative detection of faster-than-Nyquist signaling," IEEE Trans. Veh. Technol., vol. 64, no. 5, pp. 2122-2128, May 2014.

[16] T. Ishihara, S. Sugiura, and L. Hanzo, "The evolution of faster-thanNyquist signaling," to appear in IEEE Access, 2021.

[17] I. Kanaras, A. Chorti, M. R. Rodrigues, and I. Darwazeh, "Spectrally efficient FDM signals: Bandwidth gain at the expense of receiver complexity," in IEEE Int. Conf. Commun., 2009, pp. 1-6.

[18] I. Darwazeh, T. Xu, T. Gui, Y. Bao, and Z. Li, "Optical SEFDM system; bandwidth saving using non-orthogonal sub-carriers," IEEE Photonics Technol. Lett., vol. 26, no. 4, pp. 352-355, 2013.

[19] S. Li, B. Bai, J. Zhou, Q. He, and Q. Li, "Superposition coded modulation based faster-than-Nyquist signaling," Wireless Commun. Mobile Comput., vol. 2018, 2018.

[20] F. Rusek and J. B. Anderson, "Constrained capacities for faster-thanNyquist signaling," IEEE Trans. Inf. Theory, vol. 55, no. 2, pp. 764-775, Feb. 2009.

[21] L. Dai, B. Wang, Z. Ding, Z. Wang, S. Chen, and L. Hanzo, "A survey of non-orthogonal multiple access for 5G," IEEE Commun. Sur. \& Tut., vol. 20, no. 3, pp. 2294-2323, May 2018.

[22] R. M. Gray, Toeplitz and Circulant Matrices: A Review. Now Foundations and Trends, 2006.

[23] Z. Ding, Z. Yang, P. Fan, and H. V. Poor, "On the performance of non-orthogonal multiple access in $5 \mathrm{G}$ systems with randomly deployed users," IEEE Signal Process. Lett., vol. 21, no. 12, pp. 1501-1505, 2014.

[24] S. Li, B. Bai, J. Zhou, P. Chen, and Z. Yu, "Reduced-complexity equalization for faster-than-Nyquist signaling: New methods based on Ungerboeck observation model," IEEE Trans. Commun., vol. 66, no. 3, pp. 1190-1204, Mar. 2017.

[25] S. Li, W. Yuan, J. Yuan, B. Bai, D. W. K. Ng, and L. Hanzo, "Timedomain vs. frequency-domain equalization for FTN signaling," IEEE Trans. Veh. Technol., vol. 69, no. 8, pp. 9174-9179, Jun. 2020.

[26] Z. Wei, D. W. K. Ng, J. Yuan, and H. Wang, "Optimal resource allocation for power-efficient MC-NOMA with imperfect channel state information," IEEE Trans. Commun., vol. 65, no. 9, pp. 3944-3961, May 2017.

[27] Z. Wei, D. W. K. Ng, and J. Yuan, "NOMA for hybrid mmWave communication systems with beamwidth control," IEEE J. Sel. Topics Signal Process., vol. 13, no. 3, pp. 567-583, Jun. 2019.

[28] W. Yuan, N. Wu, J. Yuan, D. W. K. Ng, and L. Hanzo, "Joint data and active user detection for grant-free FTN-NOMA in dynamic networks," in IEEE Int. Conf. Commun., 2020, pp. 1-6.

[29] W. Yuan, N. Wu, A. Zhang, X. Huang, Y. Li, and L. Hanzo, "Iterative receiver design for FTN signaling aided sparse code multiple access," IEEE Trans. Wireless Commun., vol. 19, no. 2, pp. 915-928, Nov. 2020.

[30] B. Simon, Szegö's Theorem and Its Descendants: Spectral Theory for L2 Perturbations of Orthogonal Polynomials. Princeton university press, 2010, vol. 6.

[31] M. Qiu, Y.-C. Huang, and J. Yuan, "Downlink non-orthogonal multiple access without SIC for block fading channels: An algebraic rotation approach," IEEE Trans. Wireless Commun., vol. 18, no. 8, pp. 39033918, Jun. 2019.

[32] M. Qiu, Y.-C. Huang, J. Yuan, and C.-L. Wang, "Lattice-partition-based downlink non-orthogonal multiple access without SIC for slow fading channels," IEEE Trans. Commun., vol. 67, no. 2, pp. 1166-1181, Oct. 2018.

[33] Y. J. D. Kim, "Properties of faster-than-Nyquist channel matrices and folded-spectrum, and their applications," in Proc. IEEE Wireless Commun. Net. Conf., 2016, pp. 1-7.

[34] Y. J. D. Kim, J. Bajcsy, and D. Vargas, "Faster-than-Nyquist broadcasting in Gaussian channels: Achievable rate regions and coding," IEEE Trans. Commun., vol. 64, no. 3, pp. 1016-1030, 2016.

[35] F. Rusek and A. Prlja, "Optimal channel shortening for MIMO and ISI channels," IEEE Trans. Wireless Commun., vol. 11, no. 2, pp. 810-818, Dec. 2011.

[36] A. T. Abebe and C. G. Kang, "Iterative decoders for FTN-based NOMA scheme to multiplex sporadic and broadband transmission," in Int. Conf. Inf. Commun. Techn. Conv. (ICTC), 2018, pp. 813-817.

[37] T. M. Cover and J. A. Thomas, Elements of Information Theory. John Wiley \& Sons, 2012.
[38] B. Clerckx, Y. Mao, R. Schober, E. A. Jorswieck, D. J. Love, J. Yuan, L. Hanzo, G. Y. Li, E. G. Larsson, and G. Caire, "Is NOMA efficient in multi-antenna networks? A critical look at next generation multiple access techniques," IEEE Open J. Commun. Soc., vol. 2, pp. 1310-1343, Jun. 2021

[39] F. R. Gantmakher, The Theory of Matrices. American Mathematical Soc., 1959, vol. 131.

[40] P. J. Brockwell, R. A. Davis, and S. E. Fienberg, Time Series: Theory and Methods. Springer Science \& Business Media, 1991.

[41] W. Rudin, Real and Complex Analysis. Tata McGraw-hill education, 2006.

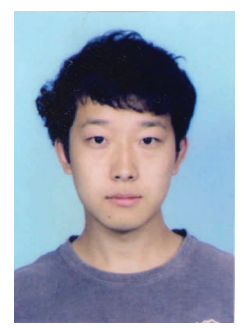

Shuangyang Li (S'18) received the B.S., M.S., and $\mathrm{Ph}$. D. degrees from Xidian University, China, in 2013, 2016, and 2021, respectively. He is currently pursuing the Ph.D. degree in the University of New South Wales. He was the TPC Co-Chair of the IEEE International Conference on Communications in China (ICCC) 2021 Workshop on orthogonal time frequency space (OTFS). He is a founding member and the secretary of the special interest group (SIG) on OTFS. He was a recipient of the 2021 student travel grant of IEEE International Conference on Communications (ICC). His research interests include signal processing, channel coding and their applications to communication systems, with a specific focus on waveform designs.

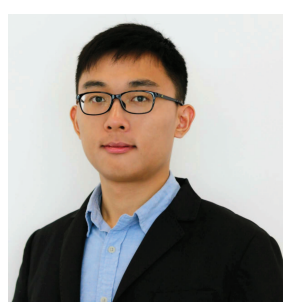

Zhiqiang Wei (S'16-M'19) received the B.E. degree in information engineering from Northwestern Polytechnical University (NPU), Xi'an, China, in 2012, and the Ph.D. degree in electrical engineering and telecommunications from the University of New South Wales (UNSW), Sydney, Australia, in 2019. From 2019 to 2020, he was a Postdoctoral Research Fellow with UNSW. He is currently a Humboldt Postdoctoral Research Fellow with the Institute for Digital Communications, Friedrich-Alexander University Erlangen-Nuremberg (FAU), Erlangen, Germany. He received the Best Paper Awards at the IEEE International Conference on Communications (ICC), 2018. He is the founding co-chair (publications) of the IEEE ComSoc special interest group on OTFS (OTFSSIG). He is serving as a Guest Editor of the Frontiers in Signal Processing Special Issue on Orthogonal Time Frequency Space Modulation for 6G Communications. He has been serving as the TPC Co-Chair of the IEEE ICC 2021 Workshop on OTFS, the IEEE ICC 2022 Workshop on OTFS, and IEEE International Conference on Communications in China (ICCC) 2021 Workshop on OTFS. He was recognized as an Exemplary Reviewer of IEEE Transactions on Communications in 2017-2020 and IEEE Transactions on Wireless Communications in 2017 and 2018. 


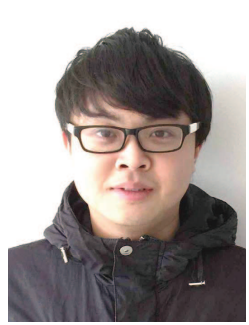

Weijie Yuan (S'15-M'19) received the B. E. degree from the Beijing Institute of Technology, China, and the Ph.D. degree from the University of Technology Sydney, Australia, in 2013 and 2019, respectively. From 2019 to 2021, he was a Research Associate with the University of New South Wales. He is currently an assistant professor with the Department of Electrical and Electronic Engineering, Shenzhen, China. He has served as a Research Assistant with the University of Sydney, a Visiting Associate Fellow with the University of Wollongong, and a Visiting Fellow with the University of Southampton, from 2017 to 2019. In 2016, he was a Visiting Ph.D. Student with the Institute of Telecommunications, Vienna University of Technology, Austria. He is the founding chair of the IEEE ComSoc special interest group on orthogonal time frequency space (OTFS-SIG). He has served as the Co-Chair and Co-Organizer for workshops and special sessions in IEEE flagship conferences, including ICC, ICCC, SPAWC, VTC, WCNC, and ICASSP. He is currently serving as an Associate Editor for the IEEE Communications Letters and EURASIP Journal on Advances in Signal Processing, the Lead Guest Editor for two special issues in IEEE Transactions on Green Communications and China Communications. His research interests including statistical inference, OTFS, and integrated sensing and communication (ISAC).

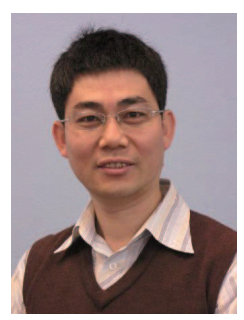

Jinhong Yuan (M'02-SM'11-F'16) received the B.E. and Ph.D. degrees in electronics engineering from the Beijing Institute of Technology, Beijing, China, in 1991 and 1997, respectively. From 1997 to 1999 , he was a Research Fellow with the School of Electrical Engineering, University of Sydney, Sydney, Australia. In 2000, he joined the School of Electrical Engineering and Telecommunications, University of New South Wales, Sydney, Australia, where he is currently a Professor and Head of Telecommunication Group with the School. He has published two books, five book chapters, over 300 papers in telecommunications journals and conference proceedings, and 50 industrial reports. $\mathrm{He}$ is a co-inventor of one patent on MIMO systems and two patents on lowdensity-parity-check codes. He has co-authored four Best Paper Awards and one Best Poster Award, including the Best Paper Award from the IEEE International Conference on Communications, Kansas City, USA, in 2018, the Best Paper Award from IEEE Wireless Communications and Networking Conference, Cancun, Mexico, in 2011, and the Best Paper Award from the IEEE International Symposium on Wireless Communications Systems, Trondheim, Norway, in 2007. He is an IEEE Fellow and currently serving as an Associate Editor for the IEEE Transactions on Wireless Communications. He served as the IEEE NSW Chapter Chair of Joint Communications/Signal Processions/Ocean Engineering Chapter during 2011-2014 and served as an Associate Editor for the IEEE Transactions on Communications during 2012-2017. His current research interests include error control coding and information theory, communication theory, and wireless communications.

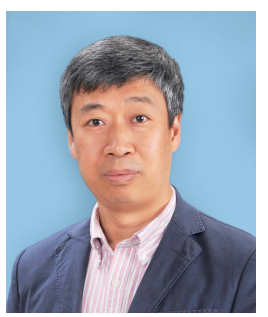

Baoming Bai (S'98-M'00-SM'18) received the B.S. degree from the Northwest Telecommunications Engineering Institute, China, in 1987, and the M.S. and $\mathrm{Ph} . \mathrm{D}$. degrees in communication engineering from Xidian University, China, in 1990 and 2000, respectively. From 2000 to 2003, he was a Senior Research Assistant at the Department of Electronic Engineering, City University of Hong Kong. Since April 2003, he has been with the State Key Laboratory of Integrated Services Networks (ISN), School of Telecommunication Engineering, Xidian University, China, where he is currently a Professor. In 2005, he was with the University of California, Davis, CA, USA, as a Visiting Scholar. In 2018, he spent one month as a Senior Visiting Fellow at McMaster University, Ontario, Canada. Dr. Bai co-authored the book Channel Coding for 5G (in Chinese, 2020). His research interests include information theory and channel coding, wireless communication, and quantum communication.

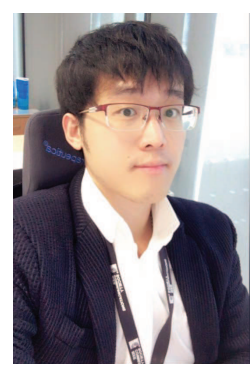

Derrick Wing Kwan Ng (S'06-M'12-SM'17-F'21) received the bachelor degree with first-class honors and the Master of Philosophy (M.Phil.) degree in electronic engineering from the Hong Kong University of Science and Technology (HKUST) in 2006 and 2008, respectively. He received his Ph.D. degree from the University of British Columbia (UBC) in Nov. 2012. He was a senior postdoctoral fellow at the Institute for Digital Communications, Friedrich-Alexander-University ErlangenNürnberg (FAU), Germany. He is now working as Scientia Associate Professor at the University of New South Wales, Sydney, Australia. His research interests include convex and non-convex optimization, physical layer security, IRS-assisted communication, UAV-assisted communication, wireless information and power transfer, and green (energy-efficient) wireless communications.

Dr. Ng has been listed as a Highly Cited Researcher by Clarivate Analytics since 2018. He received the Australian Research Council (ARC) Discovery Early Career Researcher Award 2017, the Best Paper Awards at the WCSP 2020, 2021, IEEE TCGCC Best Journal Paper Award 2018, INISCOM 2018 , IEEE International Conference on Communications (ICC) 2018, 2021, IEEE International Conference on Computing, Networking and Communications (ICNC) 2016, IEEE Wireless Communications and Networking Conference (WCNC) 2012, the IEEE Global Telecommunication Conference (Globecom) 2011, and the IEEE Third International Conference on Communications and Networking in China 2008. He has been serving as an editorial assistant to the Editor-in-Chief of the IEEE Transactions on Communications from Jan. 2012 to Dec. 2019. He is now serving as an editor for the IEEE Transactions on Communications, the IEEE Transactions on Wireless Communications, and an area editor for the IEEE Open Journal of the Communications Society.

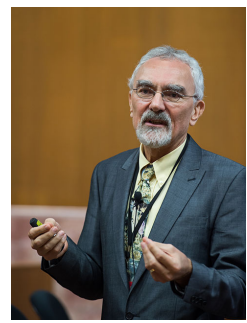

Lajos Hanzo (http://www-mobile.ecs.soton.ac.uk, https://en.wikipedia.org/wiki/Lajos_Hanzo)

(FIEEE'04) received his Master degree and Doctorate in 1976 and 1983, respectively from the Technical University (TU) of Budapest. He was also awarded the Doctor of Sciences (DSc) degree by the University of Southampton (2004) and Honorary Doctorates by the TU of Budapest (2009) and by the University of Edinburgh (2015). He is a Foreign Member of the Hungarian Academy of Sciences and a former Editor-in-Chief of the IEEE Press. He has served several terms as Governor of both IEEE ComSoc and of VTS. He has published 2000+ contributions at IEEE Xplore, 19 Wiley-IEEE Press books and has helped the fast-track career of $123 \mathrm{PhD}$ students. Over 40 of them are Professors at various stages of their careers in academia and many of them are leading scientists in the wireless industry. He is also a Fellow of the Royal Academy of Engineering (FREng), of the IET and of EURASIP. He is the recipient of the 2022 Eric Sumner Field Award. 